\title{
CAMBIO DEMOGRÁFICO Y DURACIÓN DE LA VIDA ECONÓMICAMENTE ACTIVA EN LA REGIÓN DE MAGALLANES Y LA ANTÁRTICA CHILENA 1992-2013
}

RODRIGO RIVERO-CANTILLANO ${ }^{a}$

\begin{abstract}
RESUMEN
En este artículo se analizan las implicancias del cambio demográfico en la región de Magallanes y la Antártica Chilena. Se analiza la dinámica de envejecimiento de la población y su impacto sobre la fuerza de trabajo. Concretamente observamos los cambios acontecidos en la duración de la vida activa a través de los años brutos y netos de actividad económica, el patrón de ingreso y retiro de la actividad y la esperanza de vida activa de la fuerza de trabajo. Metodológicamente se requiere de la construcción de Tablas de Vida Activa para los años 1992, 2003 y 2013. Utilizamos las declaraciones de actividad e inactividad económica para la región obtenidas desde la Encuesta de Caracterización Socioeconómica Nacional (CASEN) y, por otro lado, tablas de mortalidad publicadas por el Instituto Nacional de Estadísticas (INE, 2004).
\end{abstract}

PALABRAS CLAVE: cambio demográfico, fuerza de trabajo, duración de la vida activa, Magallanes.

\section{DEMOGRAPHIC CHANGE AND THE LENGTH OF WORKING LIFE IN} THE REGION OF MAGELLAN AND CHILEAN ANTARTIC 1992-2013

\begin{abstract}
This article analyzes the implications of the demographic change in the region of Magellan and the Chilean Antarctic. It analyzes the dynamics of population aging and its impact on the labor force. In particular, it was observed the variation the working life's length through the gross and net years of economic activity, the pattern of entrance and retirement and the working life expectancy. Methodologically, we elaborated Tables of Working Life for the years 1992, 2003 and 2013. We also used information of economic activity and inactivity for the region obtained from the National Socioeconomic Characterization Survey (CASEN in spanish) and published mortality tables by National Statistics Institut (INE, 2004).
\end{abstract}

KEY WORDS: demographic change, labor force, length of working life, Magellan.

a Investigador Postdoctorado del Centro Internacional de Investigación de Historia Económica, Empresarial y de la Administración Pública (CIHEAP), adscrito a la Facultad de Administración y Economía (FAE) de la Universidad de Santiago de Chile (USACH) $\triangle$ rodrigo.rivero@usach.cl 


\section{INTRODUCCIÓN}

De la mano de las grandes transformaciones económicas y sociales por las que ha atravesado el país desde mediados del siglo XX, la población magallánica ha experimentado grandes cambios, adquiriendo las características principales con las que la conocemos hoy en día. En ese marco, la fuerza de trabajo, aquel grupo de población que se encuentra disponible para la producción de bienes y servicios, ha experimentado cambios en su tamaño, características educativas y composición por edad y sexo, características relacionadas con la participación económica, así como con la duración de la vida activa.

En perspectiva histórica, los análisis sobre el progreso económico y social suelen centrarse en variables macroeconómicas, sin embargo, uno de los cambios más significativos y que no se suele incluir en los análisis es el cambio demográfico. Estudiar el cambio demográfico en perspectiva histórica y sus efectos sobre la fuerza de trabajo resulta fundamental para comprender la dinámica del desarrollo, así como sus potencialidades (Bloom \& Williamson, 1998; Andersson, 2001; Bloom et al. 2000, 2001, 2003, 2004).

Hoy en día el envejecimiento es la dinámica demográfica dominante en la población chilena. Consiste en el cambio en la estructura etaria de la población, que conlleva a un mayor crecimiento en la proporción de la población de 65 años y más. En este contexto, la región de Magallanes y la Antártica Chilena -en adelante Magallanes-, es actualmente una de las regiones del país que lideran esta dinámica. Su nivel de envejecimiento está por sobre el promedio nacional, según la última publicación de la encuesta CASEN (2015), el 13,7\% de la población de la región correspondía a personas de 65 o más años, frente al 12,6\% del total nacional, siendo la cuarta región con mayor proporción de ancianos.

La historia de la población de Magallanes da cuenta de la singularidad de esta región en el contexto del país. Ciertamente las particularidades de la población magallánica vienen dadas por su origen. A diferencia del núcleo tradicional en el cual se ha desarrollado la población chilena desde tiempos coloniales (desde Copiapó hasta el BíoBío) sobre la base de un espontáneo mestizaje entre los españoles y pueblos originarios y desde donde han emergido los rasgos culturales típicos de la cultura mestizo-criolla, la base del poblamiento de la zona de Magallanes es "reciente". La literatura especializada destaca a la década de 1840 como el comienzo del proceso de poblamiento con base en pequeños enclaves mineros (Martinic, 2006). Primero atraídos por el oro, y luego la explotación ovina, hicieron del territorio de Magallanes una zona habitada en su gran mayoría por hombres solteros provenientes de otras regiones del país en búsqueda de beneficio económico sin las intenciones de arraigarse en el lugar (Martinic, 2002, 2016). Como consecuencia de esta "implantación" de población y a la incompatibilidad de los modos de vida económicos de, por un lado, los occidentales $y$, por otro, los originarios, al cabo de medio siglo transcurrido del primer contacto, la población originaria había sido prácticamente exterminada, quedando como una forma de hábitat el occidental (Martinic, 2002).

Durante el siglo XX, Magallanes dejó de ser aquella región de sacrificio y pasó a ser un lugar donde asentarse. En este sentido, el papel jugado por la inmigración proveniente de Europa fue determinante, gracias a ella la región adquirió su fisonomía actual. En términos demográficos esto significó una población joven, emprendedora y capacitada que lideró el desarrollo local (Martinic, 2002; Vera, 2008). De esta manera, rasgos distintivos de la población de Magallanes cuando se le compara con el contexto nacional, son el grado de urbanización y de alfabetismo, ambos muy elevados desde muy temprano en el siglo XX (Martinic, 2002). Ya en el censo de 1940, el 77,7\% de la población de la Provincia de Magallanes -actual región de Magallanes y la Antártica chilena-, correspondía a población cuya residencia era urbana, cuando en el mismo año sólo el $52 \%$ de la población total del país residía en ciudades, siendo la tercera región en proporción de población urbana después de las provincias de Santiago y Valparaíso, las dos ciudades más preeminentes en el desarrollo histórico de Chile e incluso superando a Concepción.

Estas singularidades están estrechamente relacionadas con las dinámicas de población. Tanto la urbanización como el alfabetismo son elementos asociados a la realidad económicosocial más que demográfica, no obstante, influyen 
de forma determinante en los principales agentes de cambio demográfico, los niveles y tendencias de fecundidad y mortalidad. Además, tienen una gran influencia sobre el comportamiento de la población en edad económicamente activa (aquella entre los 15 y 64 años). Desde una perspectiva económica, el cambio demográfico, conjuntamente con los cambios en el mercado de trabajo, están detrás de las condiciones en las que se desenvuelve la relación en la oferta y demanda de trabajo, por lo que es sumamente importante cuando abordamos las tendencias observadas en la fuerza de trabajo.

Hoy en día la duración de la vida activa de la fuerza de trabajo es un punto central en la relación entre los anhelos de desarrollo y los desafíos planteados por el proceso de envejecimiento. En este sentido, la edad se convierte en una de las variables más importantes en análisis de la realidad social y económica puesto que en ella se reflejan las transformaciones socioeconómicas de una población. Por ejemplo, entre los más importantes encontramos aspectos tales como las tendencias de fecundidad y mortalidad, leyes sobre la edad mínima de ingreso a la actividad económica y sobre la edad de retiro, las mejoras en los sistemas de educación, todos ellos factores que modifican la edad de vinculación de la población al sistema de producción (Hayward \& Grady, 1990). Por este motivo, estudiar el cambio demográfico en perspectiva histórica y los efectos del proceso de envejecimiento sobre la fuerza de trabajo de Magallanes resulta fundamental para el conocimiento de esta dinámica al interior de la región, así como de la potencialidad de desarrollo regional en el marco del cambio demográfico.

En la primera parte de este trabajo analizamos los principales agentes de cambio demográfico en Magallanes, con perspectiva histórica y comparada con el país, explicando sus causas, así como sus efectos sobre la fuerza de trabajo magallánica entre los años 1960 y 2013. En la segunda parte nos adentramos en la dinámica de la Vida Activa de la fuerza de trabajo urbana de la región. Para ello elaboramos Tablas de Vida Activa para los años 1992, 2003 y 2013, a través de las cuales conoceremos los cambios en la duración de la vida activa en el contexto de envejecimiento demográfico. Sin duda que las características distintivas de la población magallánica, hacen que sea pertinente y relevante analizar las dinámicas históricas por las que ha atravesado la población chilena en perspectiva magallánica, con el fin de comprobar los distintos comportamientos y comprender su estado actual.

\section{METODOLOGÍA}

Con el objetivo de conocer los cambios en la duración de la vida activa de la fuerza de trabajo de la región de Magallanes y la Antártica Chilena, procedemos a la elaboración de Tablas de Vida Activa -en adelante TVA-. Existen tres familias de métodos para construir estas tablas, los que se pueden clasificar como el método de tabla de vida basada en la prevalencia observada, el método de la tabla de vida de múltiples decrementos y el método de vida de incrementos y decrementos. Entre todos estos, el más utilizado, a pesar de sus limitaciones, son las tablas de vida laboral basadas en la prevalencia observada del fenómeno que fue propuesto por Durand (1948), para comparar las expectativas de vida laboral de blancos y afroamericanos en Estados Unidos. El modelo original fue retomado por Sullivan (1971), con tanto éxito que pasó a denominarse método Sullivan. Este método ha sido y sigue siendo muy utilizado para realizar estudios comparativos de estados de actividad entre países o momentos históricos dentro de un mismo país además de otros usos (Gutiérrez de Mesa, 2010).

Por limitaciones de la información disponible, en este trabajo se elaboran TVA para los años 1992, 2003 y 2013. La imposibilidad de contar con tablas de mortalidad para cada año en que se ha publicado la encuesta CASEN, nos obliga a restringir el análisis a tres momentos en los cuales toda la información requerida se encuentra disponible (1992, 2003 y 2013). Momentos que abarcan las tres últimas décadas y nos permiten ver los cambios ocurridos con una periodicidad en torno a los diez años, periodo suficiente para analizarlos.

Siguiendo a Partida-Bush (1996), la construcción de una tabla de vida activa implica asumir una serie de supuestos fundamentales:

a) La participación de la población en la actividad económica se da dentro de un intervalo de edad previamente definido como la 
edad de ingreso -que puede ser la edad legal o una cercana a ella- y retiro $(a, d)$. Estas edades pueden variar de país en país o incluso dentro de un mismo país en dos momentos históricos. Fuera de ese intervalo la actividad es nula.

b) El ingreso a la actividad económica se da sólo cuando sus edades están comprendidas en un intervalo $(a, b)$, de menor duración que la vida activa; donde $b$ corresponde a la edad en la que la participación económica alcanza su mayor proporción. Además, dentro de este intervalo sólo se producen retiros por efecto de la mortalidad.

c) Los retiros de la actividad económica se producen en el intervalo de edades $(b, d)$. El punto $d$ se define arbitrariamente, variando de acuerdo al nivel de desarrollo y del tipo de población que se estudie. Por lo que tiende a ser menor cuanto más alto sea el nivel de desarrollo del país analizado ${ }^{1}$. En este intervalo no hay ingresos a la actividad económica.

d) Por último, se considera que la mortalidad de la población activa $e$ inactiva es igual, debido a la falta de información adecuada. Este último supuesto puede ser entendido como una restricción.

La interpretación de la Esperanza de Vida Activa por este método y, por tanto, de la tabla de vida activa es simple, representa el número medio de años que permanecería en la condición de activo o inactivo un individuo de una generación ficticia que a lo largo de su vida experimentará las mismas condiciones de actividad y mortalidad que el conjunto de la población real en el periodo de referencia (Gutiérrez de Mesa, 2010). En síntesis, la tabla de vida activa refleja en esencia la dinámica de participación de una cohorte hipotética, sometida durante toda su vida a las condiciones de mortalidad y participación implícita en las funciones utilizadas.

La ecuación compensadora a la cohorte de la tabla de vida activa sería:

Donde $l_{x}^{a}$ corresponde a los sobrevivientes activos y $l_{x}^{i}$ a los sobrevivientes inactivos. Siendo: ${ }_{n} H_{x}^{i a}$ los ingresos a la actividad, ${ }_{n} H_{x}^{a i}$ los retiros, ${ }_{n} d_{x}^{a}$

1 En nuestro caso, pese a que la edad legal a partir de la cual los sistemas de seguridad social en Chile permiten acceder a prestaciones por vejez entre las que se encuentra la jubi- las defunciones de activos y ${ }_{n} d_{x}^{i}$ las de inactivos (Partida-Bush, 1996).

\section{FUENTES}

Un requisito para la elaboración de las TVA es contar con tablas de mortalidad, las cuales las obtendremos del Instituto Nacional de Estadísticas (INE, 2004), donde se publican las tablas abreviadas de mortalidad para los periodos siguientes: 1960$61,1969-70,1980-85,1991-92$ y 2001-02, a nivel nacional y regional. Estas tablas fueron elaboradas sobre la base de una revisión crítica de los datos disponibles para su elaboración, en cuanto a la mortalidad, los registros de nacimientos y poblaciones censadas, además de una revisión bibliográfica exhaustiva de la bibliografía sobre el tema. Adicionalmente, para 2013 se recurrió a la elaboración propia de tablas de mortalidad por sexo utilizando los datos de defunciones por edad y sexo para la Región de Magallanes y la Antártica Chilena, publicados por el Institucional de Estadísticas en el Anuario de Estadísticas Vitales (2013), así como las estimaciones de población regional publicadas por el mismo organismo para el mismo año.

Desde las tablas de mortalidad obtendremos las siguientes funciones: Probabilidades de muerte $\left({ }_{n} q_{x}\right)$, Sobrevivientes de la cohorte ficticia (1 $\left.1_{x}\right)$, Años personas vividos $\left(L_{n}\right)$, Sobrevivencia $\left({ }_{n} p_{x}\right)$, Defunciones $\left({ }_{n} d_{x}\right)$ y la Esperanza de Vida $\left(e_{x}\right)$, tanto para hombres como para mujeres. El registro de una tabla de mortalidad o una tabla de vida, corresponde a un documento histórico que refleja indirectamente el nivel de vida de una época determinada, y aunque tiene un origen demográfico, su conocimiento interesa a un amplio conglomerado de investigaciones en ciencias sociales, desde el sector de la salud hasta economistas, siendo el soporte de una gran variedad de temas relacionados con la población (INE, 2004).

Por otro lado, para analizar la población económicamente activa -en adelante PEA- se disponen de los censos de población, encuestas de hogares por muestreo y en los desgloses de los censos económicos en establecimientos industriales, comerciales y de servicios, estos últimos con grandes limitaciones. De esta manera, dada la inexistencia

lación, hemos establecido como punto $d$ al grupo de 80 y más años, con la finalidad de conocer las condiciones de retiro tras la edad límite legal. 
de recuentos de población posteriores a 2002, recurriremos a la Encuesta de Caracterización Socioeconómica Nacional (CASEN), elaborada por el Ministerio de Desarrollo Social y publicada entre 1990 y 2015 . Sobre la base de las encuestas de 1992, 2003 y 2013, obtenemos un total de 101.970 registros a partir de los cuales determinamos los patrones de actividad e inactividad de la población de Magallanes. De esta manera, a partir de esta encuesta obtenemos un componente esencial, como son las tasas de participación económica de la fuerza de trabajo magallánica siguiendo la definición establecida en la encuesta, es decir, la proporción de población del país en edad laboral, que participa activamente en el mercado laboral, ya sea trabajando o buscando trabajo? .

AGENTES DE CAMBIO DEMOGRÁFICO EN LA REGIÓN DE MAGALLANES Y LA ANTÁRTICA CHILENA DENTRO DEL CONTEXTO DE LA TRANSICIÓN DEMOGRÁFICA EN CHILE: UNA MIRADA COMPARADA DE LARGO PLAZO

Las tendencias y la evolución de los dos agentes principales de cambio demográfico, mortalidad y fecundidad, se encuentran en todo momento y lugar, influidas por las circunstancias sociales y económicas en las que se desenvuelven (Livi-Bacci, 2012). En este sentido, los cambios en las estrategias de desarrollo, con la consecuente rearticulación de las estructuras productivas y sociales, un creciente rol intermediario $y$ redistributivo del Estado y la acentuación de la urbanización, constituyen el marco en el que se desarrollan las fuerzas impulsoras del cambio demográfico en Chile durante el siglo XX (Hernández, 1994).

Históricamente el patrón de mortalidad de la población magallánica se ha visto influenciado por las características sociales, económicas y ecológicas del proceso de colonización. Al tratarse de una población joven y de reciente formación, durante la primera mitad del siglo XX, las tasas de mortalidad son en su conjunto inferiores a las exhibidas por la población chilena en general (García-Moro et al. 2015). De esta manera, será con el asentamiento definitivo de la población a mediados del siglo XX, cuando observamos una convergencia entre ambos patrones, igualándose en la década de 1970 (Hernández, 1998; Martinic, 2002) (Fig. 1).

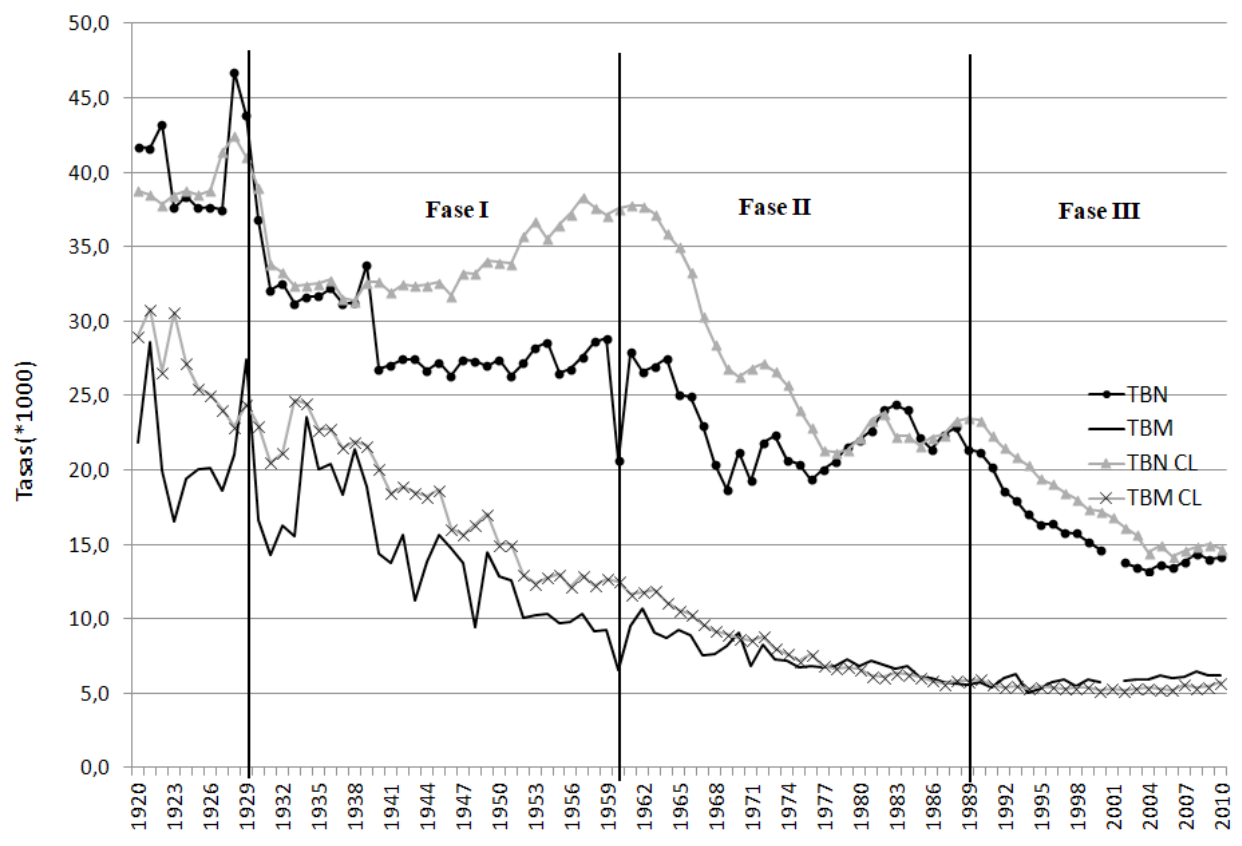

Fig. 1. Tasas brutas de natalidad y mortalidad, Chile y Magallanes 1920-2010 (por mil habitantes). Fuente: elaboración propia a base de Anuarios Estadísticos (1920-1951), Anuarios de Demografía (1952-1999) y Anuarios de Estadísticas Vitales (2000-2010).

2 http://observatorio.ministeriodesarrollosocial.gob.cl/casen/casen_def_trabajo.php 
Como podemos observar en el gráfico 1, a pesar de la excepcionalidad que caracteriza al patrón de poblamiento magallánico en el escenario nacional, las tendencias demográficas se enmarcan dentro del patrón transicional seguido por la población chilena, aunque con matices. En este sentido, la primera etapa de esta transición hacia un régimen moderno de crecimiento demográfico comienza con el declive de la mortalidad en la década de 1930. Tras tres décadas de continuo retroceso en las tasas de mortalidad, en la década de 1960 comienza la segunda etapa, cuando al declive de la mortalidad se suma el declive de la fecundidad. $\mathrm{Al}$ igual que en el global nacional, en Magallanes el descenso de la fecundidad fue rápido, reduciéndose en un $42 \%$ entre 1965 y 2005, tal como se aprecia en la tasa bruta de natalidad -en adelante TBNpasó de los 25 nacimientos por cada mil habitantes a 14,1 respectivamente, un patrón de reducción típico de los países en desarrollo, que entraron en la dinámica de la transición demográfica -en adelante TD- durante el siglo XX (Reher, 1994, 2004). Por último, la tercera etapa, vigente en la actualidad, comienza en la década de 1990 cuando ambos indicadores, mortalidad y fecundidad, alcanzan bajos niveles y profundizan su declive hacia el siglo XXI.

\section{El aumento de las expectativas de vida}

Las tasas de mortalidad y la esperanza de vida son dimensiones complementarias del bienestar de una población determinada, pues la cantidad de años que todo individuo tiene derecho a esperar vivir está en relación íntima con la mortalidad de la época, y a su vez ésta se encuentra estrechamente relacionada con las condiciones de vida que, a modo general, enfrenta la población. En este sentido, desde el comienzo de la transición de mortalidad, el descenso de las muertes entre los recién nacidos elevó considerablemente la esperanza de vida al nacer. García-Moro et al. (2015), cifran la esperanza de vida al nacer en la región de Magallanes en la primera década del siglo XX en los 40,9 años para los hombres y 44,2 para las mujeres. En la década de 1960, los años que los magallánicos esperaban vivir alcanzó los 63,4 entre los hombres y 66,2 en las mujeres, mientras que al comienzo del siglo XXI alcanzó los 72,1 y 79,4 respectivamente, los logros en este sentido han sido notables (Tabla 1).
Tabla 1. Años de esperanza de vida al nacer $\left(e_{0}\right)$. Magallanes 1960-2014.

\begin{tabular}{cccc}
\hline \multicolumn{4}{c}{ Esperanza de vida al nacer $\left(e_{0}\right)$} \\
\hline Periodo & Ambos sexos & Hombres & Mujeres \\
\hline $1960-1961$ & 63,37 & 60,71 & 66,13 \\
$1969-1970$ & 64,56 & 61,96 & 67,26 \\
$1980-1985$ & 71,14 & 67,75 & 74,67 \\
$1991-1992$ & 74,60 & 70,90 & 78,45 \\
$2001-2002$ & 75,73 & 72,21 & 79,39 \\
$2013-2014^{*}$ & 77,92 & 74,44 & 81,85 \\
\hline
\end{tabular}

Fuente: INE (2004). "Elaboración propia a base de INE (2013, 2014)

Como resultado, del proceso de modernización social y económica, el rápido declive de la mortalidad ha tenido un papel destacado en el cambio demográfico, pues primero impacta en mayores oportunidades de sobrevivencia de los neonatos, y posteriormente con la prolongación de la vida a edades avanzadas (Galleguillos \& Sierralta, 1989; Medina \& Kaempffer, 2000; Behm, 2014). Desde un punto de vista biológico, la transición de mortalidad se dio mediante la transición epidemiológica que cambió profundamente los patrones de morbilidad y mortalidad de la población, transitando de un escenario de alta prevalencia de enfermedades infecciosas a otro donde la mortalidad general se produce mayoritariamente por efecto de enfermedades crónico-degenerativas (Szotz-Mesa, 2003; Villa, 2004; García et al. 2015). De esta forma, en el largo plazo los cambios en el perfil de los fallecimientos influyen sobre la estructura de edad de la población de Magallanes (Tabla 2).

La descomposición de la esperanza de vida por edades nos revela cómo contribuyen las mejoras de las expectativas de vida a distintas edades al mejoramiento de la esperanza de vida total. Como puede observarse en el cuadro XX, el patrón de ganancias de esperanza de vida por edades ha cambiado considerablemente a lo largo del tiempo, pasando desde un esquema dominado principalmente por la mortalidad infantil hacia otro en que las contribuciones se concentran en edades avanzadas (Fig. 2).

Hasta la década de 1980, los mayores aportes en años al aumento de la esperanza de vida provenían de los más jóvenes. La rápida reducción de las tasas de mortalidad, pero sobre 
Tabla 2. Años de esperanza de vida a diferentes edades, región de Magallanes y la Antártica Chilena, 1960-2014.

\begin{tabular}{|c|c|c|c|c|c|c|c|c|c|}
\hline & & $\begin{array}{c}1960- \\
1961\end{array}$ & $\begin{array}{l}1969- \\
1970\end{array}$ & $\begin{array}{c}1980- \\
1985\end{array}$ & $\begin{array}{c}1991- \\
1992\end{array}$ & $\begin{array}{c}2001- \\
2002\end{array}$ & $\begin{array}{l}2013- \\
2014^{*}\end{array}$ & Dif. Abs & Dif. Rel \% \\
\hline \multirow[t]{4}{*}{$e_{0}$} & Hombre & 60,7 & 62,0 & 67,8 & 70,9 & 71,2 & 74,4 & 13,7 & 22,6 \\
\hline & Mujer & 66,1 & 67,3 & 74,7 & 78,5 & 79,4 & 81,9 & 15,7 & 23,8 \\
\hline & Dif. Abs. & 5,4 & 5,3 & 6,9 & 7,6 & 8,2 & 7,4 & 2,0 & 36,7 \\
\hline & Dif. Rel. \% & 8,9 & 8,6 & 10,2 & 10,6 & 11,5 & 10,0 & & \\
\hline \multirow[t]{4}{*}{$e_{40}$} & Hombre & 30,2 & 30,2 & 31,7 & 34,1 & 34,8 & 36,6 & 6,4 & 21,4 \\
\hline & Mujer & 33,7 & 34,0 & 37,6 & 40,4 & 40,7 & 43,1 & 9,4 & 27,9 \\
\hline & Dif. Abs. & 3,5 & 3,8 & 5,9 & 6,3 & 5,9 & 6,5 & 2,9 & 83,8 \\
\hline & Dif. Rel. \% & 11,6 & 12,7 & 18,7 & 18,4 & 17,0 & 17,6 & & \\
\hline \multirow[t]{4}{*}{$e_{65}$} & Hombre & 12,4 & 12,7 & 13,2 & 14,4 & 14,7 & 15,4 & 3,0 & 24,5 \\
\hline & Mujer & 14,2 & 14,8 & 16,9 & 18,2 & 18,2 & 20,0 & 5,9 & 41,2 \\
\hline & Dif. Abs. & 1,8 & 2,1 & 3,7 & 3,8 & 3,6 & 4,6 & 2,8 & 157,0 \\
\hline & Dif. Rel. \% & 14,4 & 16,3 & 28,1 & 26,1 & 24,3 & 29,8 & & \\
\hline
\end{tabular}

Fuente: INE (2004). "Elaboración propia a base de INE (2013, 2014).

todo de las elevadas tasas de mortalidad infantil. A partir de la década de 1980 las ganancias han sido cada vez más reducidas, no obstante, lo relevante de cara al proceso de envejecimiento es el cambio en el perfil de los grupos con mayores aportaciones, que pasan primero a las edades intermedias y con la llegada del nuevo siglo, se hacen cada vez más importantes en los grupos de edades avanzadas.

Visto de este modo es fácil comprender que las expectativas de la sociedad que protagonizaron este cambio hayan crecido con la misma intensidad. Con mayores expectativas de vida, las razones para el desarrollo de proyectos personales y también colectivos también son mayores. En este sentido, las mayores expectativas de vida de la población se traducen, por un lado, en una mayor demanda por educación y capacitación, y por otro lado, en la permanencia en la actividad económica más allá de la edad legal de retiro. De esta manera la reducción de la mortalidad interviene en el volumen como en las características de la fuerza de trabajo.

\section{Cambios en la fecundidad}

En el transcurso del siglo XX y lo que va del siglo XXI, la sociedad chilena ha experimentado una transformación sustantiva en su nivel de fecundidad. Generación tras generación, el patrón reproductivo se modificó para alcanzar los típicos bajos niveles atribuibles a las sociedades más modernas y desarrolladas de hoy en día. Detrás de este proceso de "modernización reproductiva" estuvo la propagación de las nuevas prácticas y conductas de control de la fecundidad (Pardo, 1979; Elizaga, 1979; Martínez-Pizarro, 1998; Donoso, 2007; Cerda, 2008). Por otro lado, la rápida reducción en la $\mathrm{TBN}$ es un patrón típico de los países en desarrollo, que entraron en la TD durante el siglo XX. En todos los casos, la TD se inicia con la reducción de la mortalidad al que luego se adecúan los niveles de fecundidad (Reher, 2004) (Fig. 3).

Los indicadores de fecundidad generalmente pretenden enseñar el nivel y los cambios de tendencia a través del tiempo, sin embargo, las transformaciones de los patrones reproductivos requieren conocer los cambios en el número de hijos deseados, que en definitiva, es el detonante de la transición de fecundidad (Martínez-Pizarro, 1998). La Descendencia Final -en adelante DF- de las cohortes o generaciones de mujeres no solteras -aquellas mujeres que han pasado por el vínculo matrimonial o unión consensual- residentes en la Región de Magallanes y la Antártica Chilena en 

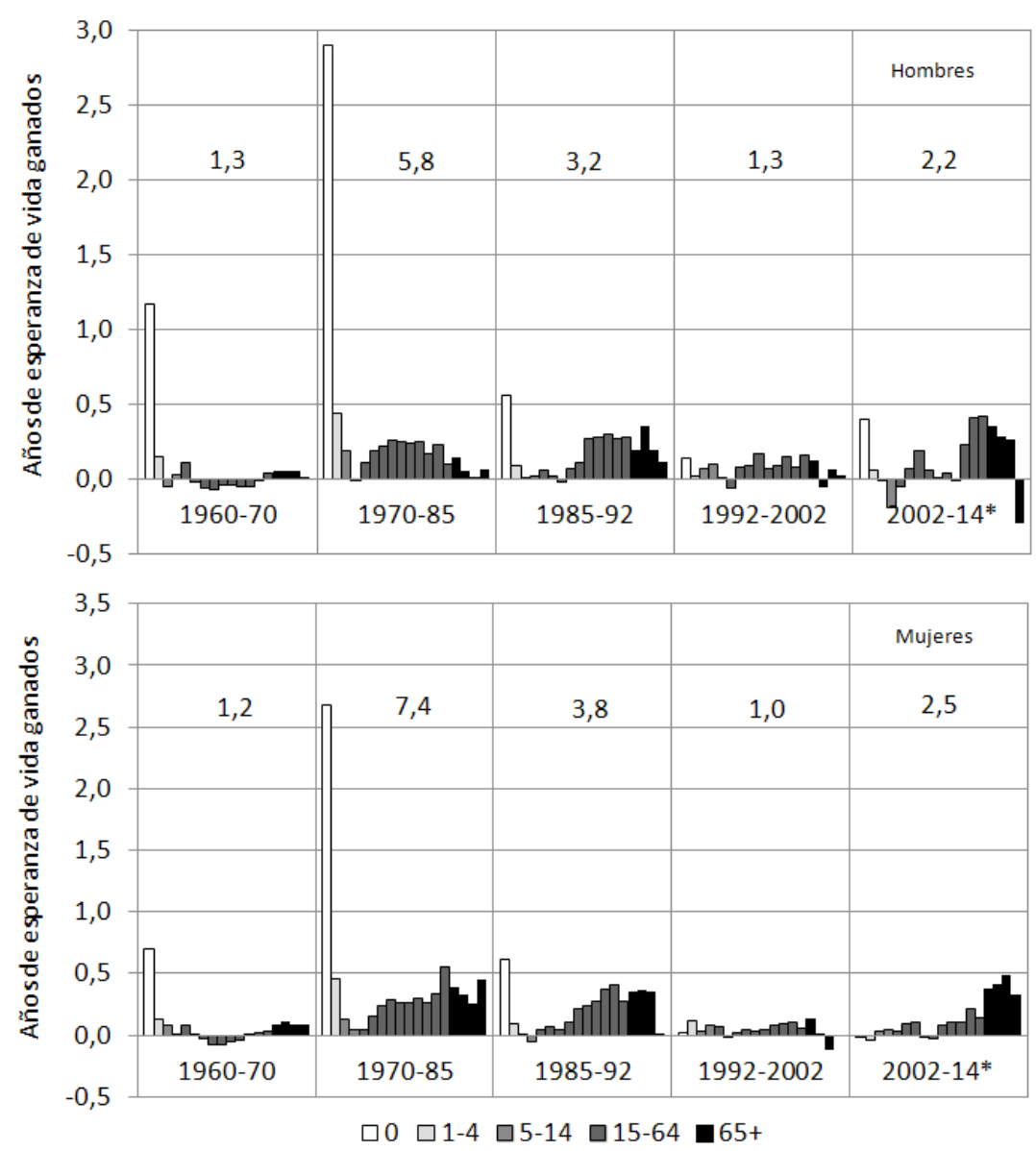

Fig. 2. Ganancias en esperanza de vida por grupos de edad y sexo (en años). Magallanes 1960-2014.

Fuente: INE (2004). *Elaboración propia a base de INE (2013, 2014).

los censos de 1982, 1992 y 2002, nacidas entre 1910 y 1960 y cuyas edades sean entre los 15 y los 45 años -edades consideradas como los límites biológicos de inicio y término del periodo reproductivo- nos permite conocer con una perspectiva longitudinal el declive de la fecundidad a través de sus periodos reproductivos que abarcan desde 1925 hasta 2009 (Tabla 3) ${ }^{3}$.

En perspectiva de largo plazo, se puede apreciar la reducción generalizada en la "intensidad" de la fecundidad a través de las sucesivas cohortes de mujeres, expresada en el total de hijos que en

3 La DF o "paridez acumulada" es un indicador que se refiere al número promedio de hijos que tendría una cohorte o generación real de mujeres al cabo del periodo reproductivo o vida fértil. Este procedimiento para calcular la DF que utiliza las probabilidades de agrandamiento de las familias permite analizar con más detalle las modalidades de constitución de promedio tiene una mujer al final de su periodo fértil, no obstante, al contrastar la DF en la región con la del conjunto del país observamos diferencias importantes. En primer lugar, se confirma el menor nivel de fecundidad de las mujeres magallánicas. Diversos estudios dan cuenta que a nivel regional, la fecundidad de Magallanes ha sido históricamente menor que el nivel del país y similar a las regiones más urbanizadas, aquellas con más de un $80 \%$ de población urbana (Martínez-Pizarro, 1998). En segundo lugar, existe un retraso, en el inicio de la tendencia de declive, mientras que en el

la descendencia y entrega una visión más próxima de cómo se producen los cambios en la fecundidad (Welti, 1997). Estudiar la fecundidad de las generaciones es especialmente apropiado para la observación y comprensión de los cambios en el comportamientos reproductivos (Devolder et al. 2006). 


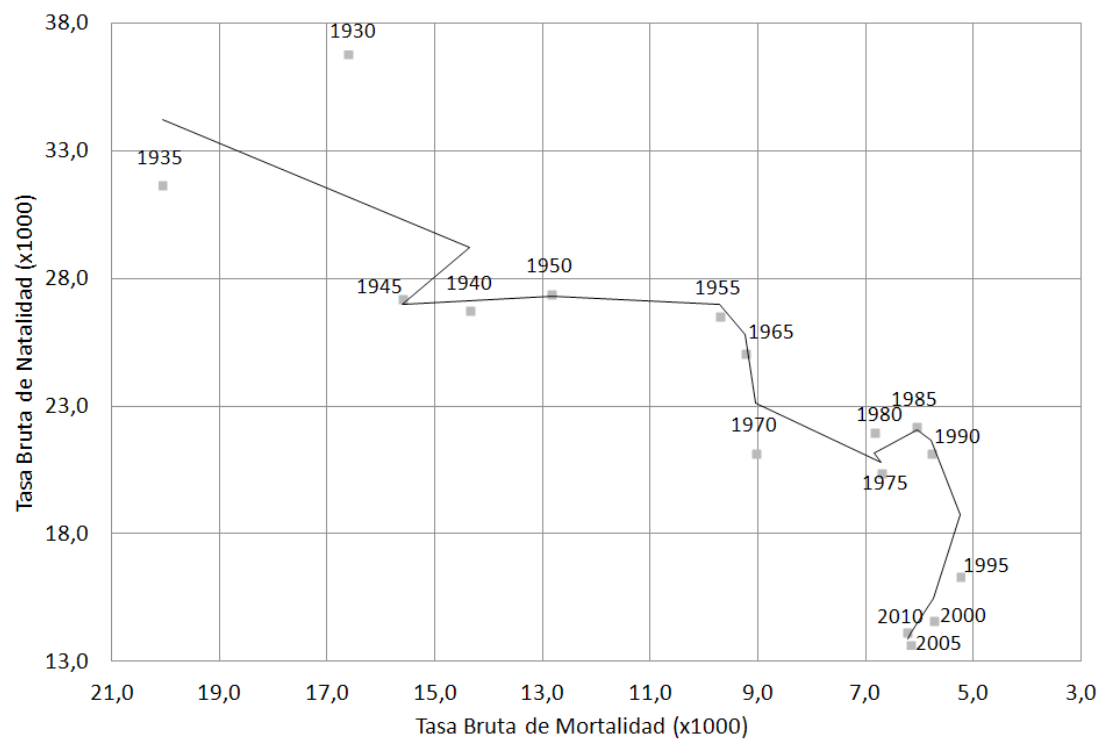

Fig. 3. Trayectorias de mortalidad y natalidad (Tasas brutas por mil habitantes), Magallanes 1930-2010. Fuente: Elaboración propia a base de Anuarios Estadísticos (1920-1951), Anuarios de Demografía (19521999) y Anuarios de Estadísticas Vitales (2000-2010).

país comienza en la cohorte 1930 , en la región el declive se consolida en la cohorte de 1940. En tercer lugar, entre las cohortes de 1910 a 1930 los patrones reproductivos divergen, mientras se observa una tendencia al alza en el conjunto del país, en Magallanes se mantiene el nivel y a partir de 1930 convergen, consolidando la tendencia en las cohortes más recientes (Fig. 4).

Tabla 3. Calendario reproductivo de las cohortes de mujeres nacidas entre 1910 y 1960.

\begin{tabular}{ccc}
\hline Cohorte & \multicolumn{2}{c}{ Periodo reproductivo } \\
\hline & Inicio & Fin \\
\hline 1910 & $1925-29$ & $1955-59$ \\
1915 & $1930-34$ & $1960-64$ \\
1920 & $1935-39$ & $1965-69$ \\
1925 & $1940-44$ & $1970-74$ \\
1930 & $1945-49$ & $1975-79$ \\
1935 & $1950-54$ & $1980-84$ \\
1940 & $1955-59$ & $1985-89$ \\
1945 & $1960-64$ & $1990-94$ \\
1950 & $1965-69$ & $1995-99$ \\
1955 & $1970-74$ & $2000-04$ \\
1960 & $1975-89$ & $2005-09$ \\
\hline
\end{tabular}

Fuente: Elaboración propia.
El menor nivel de DF de la región respecto del nacional, se relaciona en primer lugar, con las condiciones sociodemográficas y ecológicas propias de la región, que han influido históricamente en un patrón reproductivo caracterizado por un menor nivel de natalidad en relación al total nacional (Hernández, 2015). Los cambios de la tendencia de la fecundidad impactan de forma directa en la estructura de la población a través del ritmo de crecimiento de la población dependiente infantil. En el futuro se espera que la fecundidad de la región siga disminuyendo, intensificando el proceso de envejecimiento, y que la población de 65 años y más adquiera un mayor protagonismo en la estructura de edad de la población chilena.

\section{Envejecimiento demográfico}

Los efectos de largo plazo de las tendencias históricas de mortalidad y fecundidad tienen como resultado la modificación de la estructura por edades de la población de la región. Estos dos elementos, más las migraciones, son los componentes esenciales de la ecuación compensadora, que determina el crecimiento o retroceso de una población, pero también según sea la configuración de sus elementos determina el sentido en que cambia su estructura por edades, 


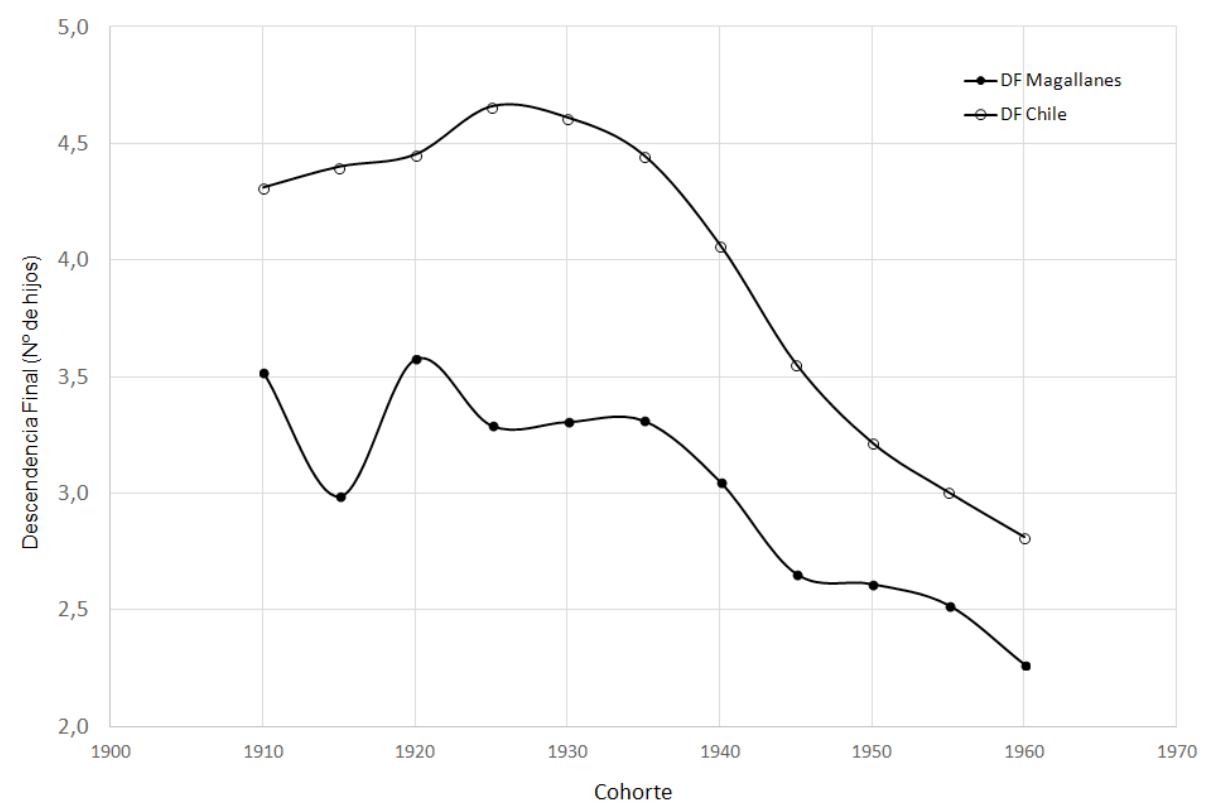

Fig. 4. Descendencia Final en Chile y Magallanes (número de hijos), cohortes de nacimiento 1910-1960.

Fuente: Elaboración propia a base microdatos censales (1960-2002), disponibles en Integrated Public Use Microdata Series (IPUMS-I).

es decir, las coyunturas de rejuvenecimiento o envejecimiento demográfico ${ }^{4}$.

La estructura por edades de una población, es uno de los temas centrales del análisis demográfico, máxime cuando a través de la gran diversidad de experiencias a nivel internacional, se ha demostrado que las transformaciones en la estructura demográfica pueden constituir retos y oportunidades importantes para el desarrollo de los países a través de las repercusiones sobre la fuerza de trabajo (Easterlin, 1978; Bloom \& Williamson, 1998; Bloom et al. 2001, 2003, 2004).

Una de las implicaciones más significativas de la TD en el largo plazo es el cambio en la relación proporcional entre los potencialmente productivos y los potencialmente dependientes, es decir, los menores de 15 años y mayores de 65, respecto del grupo de 15-64. En la Tabla 4 se aprecia cómo desde la década de 1960 la población magallánica durante la segunda etapa de la TD, comienza un proceso de envejecimiento que se traduce en la disminución en la proporción de los menores de 15 años y un aumento considerable de la población

4 La historiografía sobre la población de Magallanes da cuenta de la importancia por el proceso migratorio hacia la zona desde el inicio de su poblamiento. No obstante, ésta potencialmente productiva.

Gracias a la combinación de disminución de la fecundidad y la mayor longevidad, la estructura por edades de la población magallánica va adquiriendo paulatinamente la configuración con la que la conocemos hoy en día. A partir de la década de 1980, el grupo entre los 15 y 64 años crece hasta alcanzar el $70 \%$ de la población total en 2014. Esto es lo que se conoce como el "bono o dividendo demográfico" (Bloom et al. 2003). El bono demográfico consiste en aquellos cambios en la estructura por edades que configuran un periodo en que la proporción de personas en edades potencialmente productivas crece de manera sostenida en relación con la de personas en edades potencialmente inactivas. De esta forma las relaciones de dependencia descienden y alcanzan mínimos históricos, para posteriormente aumentar como resultado del incremento de la proporción de personas mayores.

De esta manera los cambios ocurridos en la estructura de la población magallánica resaltan a la vista al contrastar las pirámides de 1960, al inicio de la transición de fecundidad, con la pirámide de 2014.

no fue relevante durante la segunda mitad del siglo XX, al menos como para modificar la estructura demográfica de la región (ver Martinic, 2002; Ruiz, 2006) 
Tabla 4. Evolución de la estructura de la población por grupo de edad, Magallanes 1960-2014.

\begin{tabular}{ccccccc}
\hline Grupo edad & 1960 & 1970 & 1982 & 1992 & 2002 & $2014^{*}$ \\
\hline $0-14$ & 21.829 & 27.700 & 34.780 & 39.600 & 36.520 & 33.170 \\
$15-64$ & 45.982 & 54.980 & 89.480 & 96.880 & 105.480 & 114.557 \\
$65+$ & 3.320 & 5.080 & 7.280 & 8.820 & 12.060 & 16.021 \\
Total & 71.131 & 87.760 & 131.540 & 145.300 & 154.060 & 163.748 \\
$0-14(\%)$ & 30,7 & 31,6 & 26,4 & 27,3 & 23,7 & 20,3 \\
$15-64(\%)$ & 64,6 & 62,6 & 68,0 & 66,7 & 68,5 & 70,0 \\
$65+(\%)$ & 4,7 & 5,8 & 5,5 & 6,1 & 7,8 & 9,8 \\
\hline$r$ & $1960-1970$ & $1970-1982$ & $1982-1992$ & $1992-2002$ & $2002-2014$ & $1960-2014$ \\
\hline $0-14$ & 2,4 & 2,3 & 1,3 & $-0,8$ & $-1,0$ & 4,3 \\
$15-64$ & 1,8 & 5,0 & 0,8 & 0,9 & 0,8 & 9,6 \\
$65+$ & 4,3 & 3,7 & 1,9 & 3,2 & 2,9 & 17,0 \\
\hline Total & 2,1 & 4,1 & 1,0 & 0,6 & 0,6 & 8,7 \\
\hline
\end{tabular}

Fuente: Elaboración propia a base microdatos censales (1960-2002), disponibles en Integrated Public Use Microdata Series (IPUMS-I). "INE (2014).

El cambio principal se sintetiza en estrechamiento de la base de la pirámide, sobre la base de la reducción de la proporción de la población joven, menores de 25 años, siendo más notoria a menor edad. Por otro lado, se comprueba el engrosamiento de la estructura en las edades productivas y el abultamiento de los mayores de 65 años (Fig. 5).

El envejecimiento ha sido un proceso habitual en las distintas experiencias de TD, y en todas ellas se le asocia a importantes desafíos sociales y económicos, por lo que su intensidad se relaciona directamente con la magnitud de los desafíos que plantean. En el caso de Magallanes, aún podemos hablar de una intensidad moderada, dada la elevada proporción de población en edad de trabajar (entre los 15 y 64 años). Esta configuración de la estructura de la población conocida como bono demográfico u oportunidad demográfica, produce

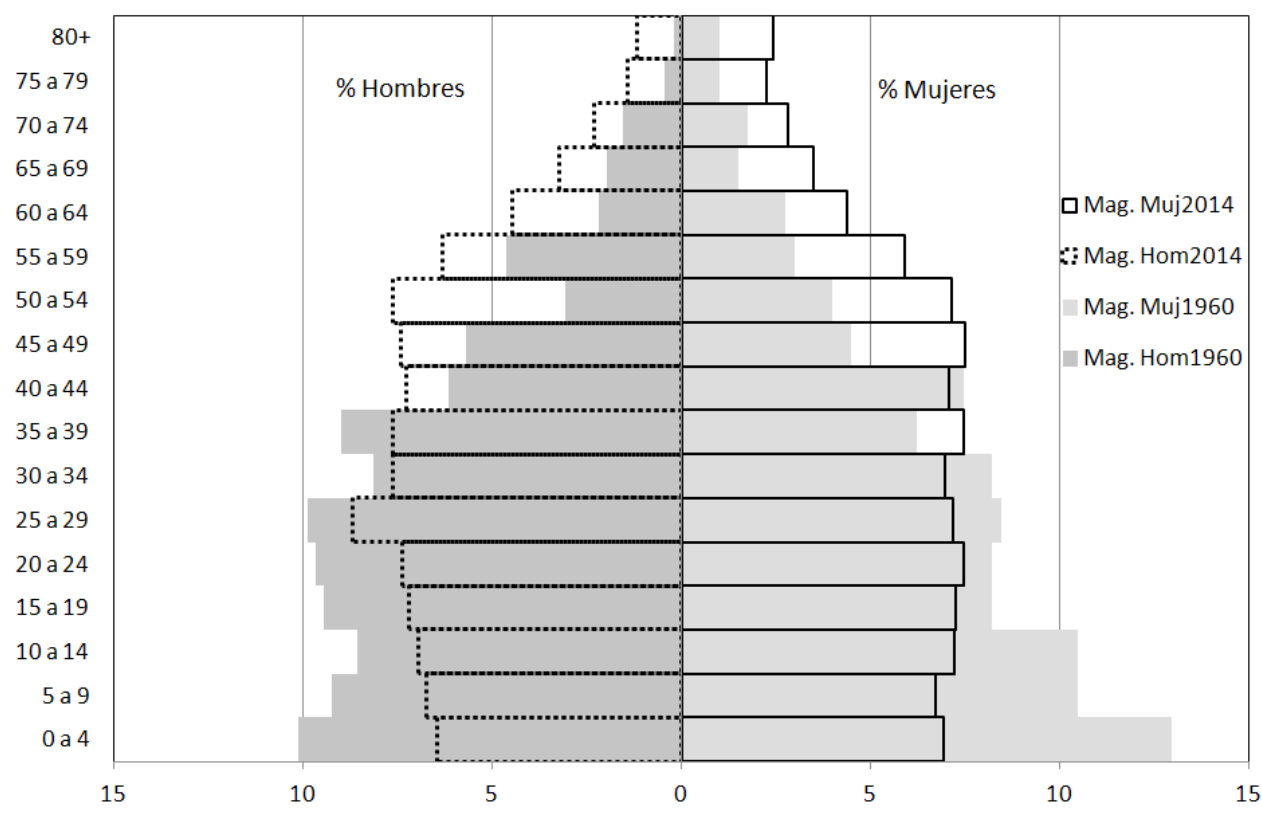

Fig. 5. Pirámide de población, Magallanes 1960-2014.

Fuente: Elaboración propia a base de INE (1964; 2014b), 
una situación particularmente favorable para el desarrollo, ya que aumentan las posibilidades de ahorro e inversión en crecimiento económico (Saad et al. 2012). No obstante, este potencial bono demográfico depende de otras variables no demográficas que deben ser propicias para su materialización. Por ejemplo, la capacidad de absorción de la mayor oferta de trabajo, de lo contrario la posibilidad de sacar provecho de una población cuya estructura se concentra en edades potencialmente productivas puede desvanecerse (Chackiel, 2004; Rosero-Bixby \& Jiménez, 2012).

\section{DINÁMICA DE LA VIDA ACTIVA DE LA FUERZA DE TRABAJO URBANA EN LA REGIÓN DE MAGALLANES Y LA ANTÁRTICA CHILENA 1992-2013}

El crecimiento en la fuerza de trabajo, la creciente participación femenina, el envejecimiento, además de la mejora en el nivel educativo, son cambios que plantean desafíos al mercado de trabajo para dar cabida a los trabajadores y las trabajadoras en condiciones óptimas de competitividad y productividad del referido factor. En este sentido, conocer los patrones de participación en la actividad económica de la población en edades potencialmente productivas nos permite evaluar el aprovechamiento de la oportunidad demográfica que implica el "bono demográfico" 5 .

En perspectiva histórica, los cambios más importantes en el patrón de participación económica por edades de la población urbana de la Región de Magallanes, se encuentran en los grupos de ingreso y de retiro de la vida activa, entre los 1519 y 20-24 años por un lado, y entre los 55-59 y 6064 años por otro. No obstante, en líneas generales, podemos observar que el declive de la participación en los grupos de entrada se ve compensado por un crecimiento generalizado de la participación en los grupos centrales de la fuerza de trabajo, aquellas edades con mayor potencial productivo, tendencia que se observa con mayor claridad desde el censo de 1992. Además, en los grupos más cercanos a

5 El grado de urbanización en la región de Magallanes y la Antártica Chilena, ha sido desde los inicios de su poblamiento muy elevado (Martinic, 2002). De esta manera en la década de 1990 la población en edad de trabajar -15 a 64 años- supera el $90 \%$ de residencia urbana con un $92,1 \%$, la edad de retiro (55-59 y 60-64), a partir de la década de los noventa, la tendencia descendente es reemplazada por crecientes tasas de participación en estos dos grupos (Tabla 5).

Tanto la postergación del ingreso al mercado de trabajo, como el aumento de la participación entre los 25 y 50 años, son dinámicas asociadas a las reformas educativas aplicadas en las décadas de 1980 y 1990, que al margen de la calidad, lograron la expansión en la cobertura educativa y el mejoramiento en el acceso a programas de capacitación y a la educación superior (Mella, 2003; Cornejo, 2006). El natural relevo generacional de las cohortes que componen la fuerza de trabajo urbana de la región, provoca un vuelco en la distribución de la fuerza de trabajo por niveles educativos. Si en 1992 tan sólo el 14,5\% de la fuerza de trabajo tenía estudios superiores y un $37 \%$ alcanzaba la educación primaria o menos, en 2013 la situación es la inversa, el 32\% contaba con estudios de nivel superior, quedando tan sólo un $17 \%$ con estudios primarios o menores. En el mismo periodo, la fuerza de trabajo con estudios de nivel medio (estudios de enseñanza media técnicos

Tabla 5. Tasas de participación económica por grupo de edad de la población urbana en edades activas, Magallanes 1960-2013.

\begin{tabular}{ccccccc}
\hline Grupo Edad & 1960 & 1970 & 1982 & 1992 & 2002 & $2013^{*}$ \\
\hline $15-19$ & 46,2 & 33,8 & 37,4 & 15,4 & 20,0 & 12,5 \\
$20-24$ & 73,8 & 62,4 & 67,5 & 60,6 & 58,1 & 45,3 \\
$25-29$ & 66,1 & 63,6 & 70,4 & 63,2 & 77,0 & 83,3 \\
$30-34$ & 63,6 & 64,1 & 69,4 & 64,0 & 74,9 & 81,8 \\
$35-39$ & 67,3 & 58,1 & 64,8 & 65,0 & 72,7 & 83,3 \\
$40-44$ & 56,5 & 61,1 & 61,1 & 61,5 & 71,6 & 78,9 \\
$45-49$ & 42,9 & 50,0 & 55,9 & 60,4 & 67,7 & 76,7 \\
$50-54$ & 52,0 & 53,2 & 49,0 & 52,8 & 61,0 & 69,7 \\
$55-59$ & 55,6 & 40,3 & 45,9 & 45,5 & 54,5 & 65,9 \\
$60-64$ & 44,4 & 37,1 & 34,5 & 35,1 & 38,8 & 56,4 \\
\hline
\end{tabular}

Fuente: Elaboración propia a base microdatos censales (19602002) disponibles en Integrated Public Use Microdata Series (IPUMS-I). *CASEN (2013).

creciendo hasta el $95,9 \%$ en 2013 . Por este motivo, los análisis se centrarán en la población activa urbana, quedando excluidas las zonas rurales y las bases antárticas, que podrían distorsionar los resultados dadas sus características de enclaves. 
o científico humanista completos o incompletos) pasó del $48 \%$ al $55 \%$. Por otro lado, es sabido que mayores niveles de estudios está altamente correlacionado con una mayor participación activa en la economía (Larrañaga, 2006) (Tabla 6).

Gracias a las mayores expectativas de vida, junto con el aumento de la cobertura del sistema educativo en la década de los noventa, la población magallánica vio aumentar sus oportunidades reales de capacitarse, reduciendo de forma considerable el riesgo de pasar a formar parte de la población económicamente activa a temprana edad, que por cierto, es uno de los prerrequisitos fundamentales para el desarrollo. Por otro lado, los estudios realizados a nivel nacional sobre los efectos de largo plazo de más años de estudios muestran el aumento en las tasas de participación a partir de los 25 años de edad de especial intensidad en las mujeres (Pardo, 1988; Paredes, 2003; Larrañaga, 2006). En Magallanes la tasa de participación femenina entre los 15 y 64 años creció desde el $36,7 \%$ en 1992 , al 53,1\% en 2013.

La educación se vincula con la participación de las mujeres en el mercado laboral a través de mejores salarios, lo cual es un incentivo de primer orden para la búsqueda de trabajo fuera del hogar, y por otro lado, un mayor nivel de educación se asocia a un menor nivel de fecundidad, lo cual facilita su entrada al mercado de trabajo (Larrañaga, 2006). En este sentido el contraste entre las pirámides demográficas de 2013 respecto de la de 1992 incluyendo los niveles de participación por cada grupo de edad, muestran una estructura de edades donde la proporción de población potencialmente productiva (entre los 15 y 64 años) es dominante y donde la participación económica de hombres y mujeres aumenta significativamente en los mayores de 40 años y sobre todo entre las mujeres, lo que da cuenta de un mayor uso y aprovechamiento, por parte del mercado de trabajo, de las potencialidades planteadas por el cambio demográfico (Fig. 6).

Respecto al aumento de la participación en edades avanzadas, este hecho está íntimamente relacionado con las mayores expectativas de vida de la población, que por necesidad o de forma voluntaria, actúan como un incentivo para la postergación del retiro de la actividad económica. Se estima que el umbral biológico del envejecimiento se encuentra en los 15 años de esperanza de vida, a partir de ahí comienza un deterioro progresivo que aleja la posibilidad de la permanencia en la actividad económica (Rivero \& Spijker, 2015). Como podemos observar en la Tabla 7 , en los años sesenta el umbral biológico de la vejez en la población de Magallanes, se encontraba en los 60,5 años en los hombres y 63,8 en las mujeres. Gracias al progreso en la esperanza de vida a edades avanzadas este umbral ha aumentado constantemente, hasta alcanzar los 65,6 años en los hombres y los 71,3 años en las mujeres durante el periodo 2013-2014.

En este contexto es natural que las tasas de participación aumenten en las edades cercanas a la edad formal de retiro, dado que las mayores expectativas de vida generan incentivos 0 necesidades para mantenerse en activo incluso una vez superados los 65 años.

Sin duda que los cambios observados en las tasas de participación económica han causado cambios importantes en la proporción de años de actividad dentro del curso de vida de las personas, que fundamentalmente se relacionan con el cambio en la edad con la que las personas inician su vida económicamente activa y la edad con la que se concluye el ciclo.

Tabla 6. Proporción de fuerza de trabajo urbana y tasas de participación por nivel educativo. Magallanes 1992-2013.

\begin{tabular}{lcccccc}
\hline & \multicolumn{3}{c}{ \% de la fuerza de trabajo } & \multicolumn{3}{c}{ Tasa de participación económica } \\
\hline Estudios & 1992 & 2003 & 2013 & 1992 & 2003 & 2013 \\
Primaria o menos & 37,1 & 22,3 & 17,2 & 53,8 & 67,4 & 63,4 \\
Secundaria & 48,4 & 55,4 & 49,6 & 55,5 & 62,9 & 62,2 \\
Superior & 14,5 & 22,0 & 32,7 & 72,2 & 74,7 & 67,1 \\
\hline
\end{tabular}

Fuente: Elaboración propia a base de CASEN (1992, 2003, 2013). 


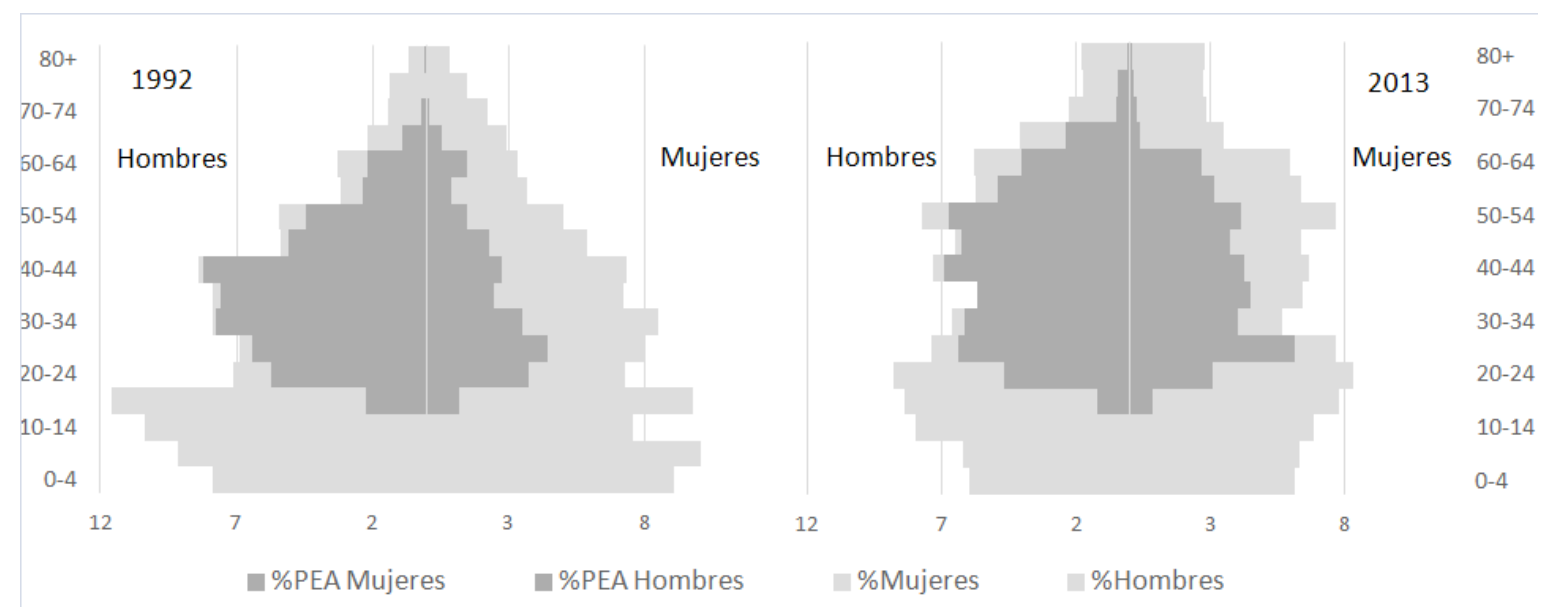

Fig. 6. Participación económica por grupos de edad población urbana. Magallanes 1992 y 2013. Fuente: Elaboración propia a base de CASEN (1992, 2003, 2013).

Tabla 7. Edad exacta a los 15 años de esperanza de vida. Magallanes 1960-2014.

\begin{tabular}{ccc}
\hline \multicolumn{3}{c}{ Umbral de envejecimiento móvil $\left(e_{\mathrm{x}}=15\right)$} \\
\hline & Hombres & Mujeres \\
\hline $1960-61$ & 60,5 & 63,8 \\
$1969-70$ & 60,9 & 64,6 \\
$1980-85$ & 61,9 & 67,8 \\
$1991-92$ & 64,1 & 69,5 \\
$2003-04$ & 64,8 & 70,0 \\
$2013-14^{*}$ & 65,6 & 71,3 \\
\hline
\end{tabular}

Fuente: INE (2004). *Elaboración propia a base de INE (2013, 2014).

\section{Patrones de Vida Activa}

\section{Duración de la vida activa}

Los años brutos y netos de vida activa sintetizan -bajo los supuestos ya señalados- el total de años que permanecería como activo un individuo de una cohorte ficticia que presentase a lo largo de su vida las mismas tasas de actividad que la población real en un momento determinado. En los años brutos se representa el número medio de años económicamente activos de trabajadores y trabajadoras que no mueren antes de la edad de retiro, mientras que los años netos incluyen la interrupción de la vida activa por la muerte antes de alcanzar la edad de retiro. En este sentido, los años brutos de vida activa dependen únicamente de la edad a la que se comienza a trabajar y de la edad de retiro, mientras que el número neto es también afectado por la mortalidad (Partida-Bush, 1996).

Teniendo en cuenta los factores que influyen en la duración de la vida activa, es de esperar que mientras mayor sea el grado de desarrollo social y económico al que se expone una población, menor será el número de años brutos de vida activa, mientras que en los años netos de vida activa, la tendencia esperada es la contraria, aumentado conforme aumenta el nivel de desarrollo, gracias a la reducción de la mortalidad, el aumento de la esperanza de vida. De esta manera, visto en perspectiva histórica los años netos de vida activa tenderán a alcanzar o igualarse a un límite superior, que son los años brutos de vida activa. Por otro lado, la diferencia entre años brutos y netos de vida activa equivale a los años de trabajo perdidos a causa de la mortalidad (Tabla 8).

$\mathrm{Al}$ estar calculados sobre la base de la prevalencia observada de las tasas de actividad e inactividad, los años brutos de vida activa reflejan las fluctuaciones en el tiempo de la participación en la fuerza de trabajo. En otras palabras, nos señalan la duración promedio de la vida o los años brutos de vida activa de un individuo tipo de una generación ficticia que experimentasen las mismas condiciones de actividad que, por ejemplo, en 1992 sería 43,5 años para hombres y 18,3 para mujeres, mientras que en 2013 sería 44,8 y 28,25 
Tabla 8. Magallanes: Años brutos y netos de vida activa a la edad de 15 años.

\begin{tabular}{ccccccc}
\hline & \multicolumn{2}{c}{ Años brutos de vida activa } & \multicolumn{2}{c}{ Años netos de vida activa } & \multicolumn{2}{c}{ Esperanza de vida $e_{15}$} \\
\hline & Hombres & Mujeres & Hombres & Mujeres & Hombres & Mujeres \\
\hline 1992 & 43,5 & 18,3 & 39,7 & 17,7 & 57,2 & 64,6 \\
2003 & 47,7 & 28,4 & 42,1 & 27,0 & 58,4 & 65,0 \\
2013 & 44,8 & 28,3 & 40,9 & 27,7 & 60,2 & 67,6 \\
\hline
\end{tabular}

Fuente: Elaboración propia.

respectivamente. Por otro lado, los años netos vida activa nos muestran el aumento real de años de vida activa gracias al retroceso de la mortalidad dentro de la población económicamente activa ${ }^{6}$. En este sentido, al comparar los años netos de vida activa al inicio de la actividad económica con los años de esperanza de vida a la misma edad -15 años-, observamos la proporción de años de actividad dentro del curso de vida de hombres y mujeres a través del tiempo, la cual disminuye levemente entre los hombres pasando del $69,4 \%$ al $68 \%$ entre 1992 y 2013 y aumenta entre las mujeres pasando del 27,3\% al 41\% entre 1992 y 2013.

La Esperanza de Vida Activa -en adelante EVA- puede entenderse como un resumen sobre el nivel de actividad que se expresa en el promedio de años en actividad para una persona, esperados bajo las condiciones de mortalidad y actividad del periodo en que se calcula, reflejando las condiciones de vida, salud, actividad y de otras dimensiones sociales del país o región en análisis 7 . Detrás de las tendencias observadas en la EVA, al igual que sucede con la esperanza de vida, están las continuas y significativas mejoras de las condiciones de salud y condiciones de vida de los trabajadores. Por otro lado, a través del tiempo, observamos la disminución de la EVA conforme avanza el curso biológico de la vida, en el mercado de trabajo de la población urbana de la Región de Magallanes y la Antártica Chilena (Tabla 9).

Observamos que entre 1992 y 2003 la EVA de la fuerza de trabajo urbana de la región, ha experimentado un aumento en las edades jóvenes

6 Los años brutos como netos de vida activa muestran fluctuaciones en las tendencias a través de los periodos que son reflejo de las tasas de participación de periodo observadas en la encuesta CASEN.

7 Como hemos señalado anteriormente respecto de la elaboración de las TVA, la EVA supone que las condiciones teóricas a las que estaría sometida una generaciones si -menores de 40 años-. Por otro lado, a partir de los 50 años de edad se observa un declive significativo de la EVA, que se hace más intensivo a partir de los 65 años, edad legal para el retiro de la fuerza de trabajo. No obstante, el avance del envejecimiento y sus efectos sobre la permanencia en la actividad económica se ven reflejados en el aumento de la EVA más allá de los 65 años, siendo más abultada en las mujeres.

Respecto de las brechas en la EVA entre hombres y mujeres éstas son más abultadas en las edades de entrada a la actividad y disminuyen a medida que se acerca la edad de retiro, e incluso superando las mujeres a los hombres después de la edad legal de retiro. En este sentido, se observa que a pesar de los obstáculos al ingreso, la fuerza de trabajo femenina, dada su mayor esperanza de vida, se mantiene en activo por mayor tiempo que los hombres, difumándose la brecha de género con el tiempo y la edad. Por otro lado, al inicio de la actividad las brechas disminuyen considerablemente entre 1992 y 2013, siendo en este último año casi un tercio de la brecha observada a principio de la década de los noventa.

\section{Transiciones entre estados}

de la vida activa e inactiva

La dinámica de la población económicamente activa está constituida por los movimientos de ingreso de trabajadores desde una edad inicial de incorporación -generalmente la mínima legal ${ }^{8}$ - hasta

el nivel de mortalidad y de participación se mantuvieran constante. Ver Elizaga (1979), Partida-Bush (1996, 2000). Su principal virtud es condensar la estructura por edad de inserción de la población en la actividad económica, así como la incidencia de la mortalidad y las transiciones entre los estados activo e inactivo a lo largo de la vida activa de una cohorte ficticia. 
Tabla 9. Magallanes. Esperanza de vida activa por grupo de edad y sexo.

\begin{tabular}{ccccccc}
\hline Grupo Edad & \multicolumn{2}{c}{1992} & \multicolumn{2}{c}{2003} & \multicolumn{2}{c}{2013} \\
\hline & Hombres & Mujeres & Hombres & Mujeres & Hombres & Mujeres \\
\hline 15 & 48,2 & 36,0 & 51,3 & 42,2 & 51,5 & 43,7 \\
20 & 43,2 & 31,0 & 46,3 & 37,2 & 46,5 & 38,7 \\
25 & 38,2 & 26,0 & 41,3 & 32,2 & 41,5 & 33,7 \\
30 & 33,3 & 23,0 & 36,3 & 27,2 & 36,5 & 28,7 \\
35 & 28,3 & 23,8 & 31,3 & 25,7 & 31,5 & 25,7 \\
40 & 23,4 & 19,8 & 27,3 & 20,7 & 26,6 & 21,8 \\
45 & 18,7 & 14,9 & 23,6 & 16,8 & 22,1 & 18,7 \\
50 & 15,1 & 11,5 & 18,6 & 15,9 & 17,8 & 15,2 \\
55 & 12,0 & 8,9 & 13,7 & 13,4 & 14,0 & 11,3 \\
60 & 8,1 & 6,2 & 10,4 & 8,4 & 10,0 & 7,2 \\
65 & 4,7 & 5,9 & 8,4 & 4,5 & 6,8 & 4,2 \\
70 & 2,2 & 1,8 & 6,5 & 7,0 & 4,0 & 6,5 \\
75 & 0,0 & 0,0 & 2,2 & 2,3 & 2,2 & 3,4 \\
$80+$ & 0,0 & 0,0 & 0,0 & 0,0 & 0,0 & 0,0 \\
\hline
\end{tabular}

la edad de participación máxima -generalmente definida por la edad legal de jubilación-. Eventualmente entre estas dos edades, se producen retiros anticipados por causa de muerte, invalidez -que puede ser momentáneo o no- o retiro voluntario, no obstante, la mejora de los niveles de mortalidad hace que los retiros por defunciones disminuyan a través del tiempo y la extensión de la esperanza de vida a edades avanzadas hace que una parte de este grupo de población permanezca voluntariamente como activa más allá de la edad legal de jubilación. Por otro lado, además de este proceso general de entradas y retiros, que ocurren principalmente en edades marginales, algunas personas reingresan después de un tiempo de inactividad. En efecto algunas personas salen de la PEA y reingresan después de un tiempo; otras salen por invalidez y en el caso de las mujeres existen causales de retiro por nupcialidad y maternidad (Elizaga, 1979).

8 La edad mínima legal para entrar al mercado de trabajo en Chile se definió sobre la base de los años de obligatoriedad de educación. No obstante, la encuesta CASEN comprende como población económicamente activa a la población de 12 o más años de edad.

9 El rápido ascenso en las tasas de retiro en el último grupo de edades (75-79) se debe a que impusimos la condición de
La gran mayoría de los que ingresan a la fuerza de trabajo son jóvenes, y a su vez, la mayor parte de los que salen de la actividad son de edad avanzada9. Por otro lado, como hemos visto, el aumento de la esperanza de vida ha llevado a un número no menos importante de trabajadores a permanecer en el mercado de trabajo aun habiendo superado la edad legal para el retiro. De esta manera, las tasas de retiro crecen hasta los 65 años y luego disminuyen influidas por la permanencia de trabajadores que pese a que cumplen la edad requerida para el retiro permanecen como activos, probablemente no gozan de una prestación económica por jubilación que les permita abandonar la actividad, por lo que deben mantenerse en la actividad algunos años más hasta que las condiciones de salud lo permitan ${ }^{10}$. Respecto de las tasas de ingreso, primero, en las mujeres nos revelan una extensión del periodo de ingreso al mercado de trabajo entre 1992 y 2013 visto a través de las edades.

participación nula a partir de los ochenta y más años.

10 Con una perspectiva de futuro, el aumento de los años de vida en inactividad, gracias al aumento de la esperanza de vida a la edad de retiro del mercado laboral, hace necesario contar con un sistema de seguridad social adecuado, que sea capaz de dar respuesta a las necesidades de las personas en su retiro (Riesco, 2006, 2009). 
En segundo lugar, al mismo tiempo que aumentan las tasas de ingreso a la actividad económica, la edad media de ingreso ha aumentado de acuerdo con la prolongación del periodo de escolaridad y formación profesional, especialmente significativo entre las mujeres. Por último, la disminución en las tasas de las edades de ingreso, luego se ve compensada con una mayor tasa de ingreso a partir de los 20 años, tanto en hombres como en mujeres, razón por la cual a través de los distintos periodos crecen las incorporaciones hasta el grupo de 30-34 años (Fig. 7).

Por otro lado, las tasas de retiro, en general, tanto en hombres como en mujeres, se caracterizan por la influencia creciente que ejerce la edad a medida que se acerca a los 65 años. En segundo lugar, llama la atención la fuerte tendencia decreciente en el retiro de las mujeres mayores de 40 años. Además de una mayor incorporación a la actividad, observamos una mayor permanencia en ella entre los 40 y 59 años. Detrás de esta tendencia puede estar un mejoramiento en las condiciones de conciliación entre el trabajo productivo y reproductivo, hecho que se ve relacionado con el mayor nivel educativo, que a su vez se relaciona con las mayores tasas de participación. También, cabe mencionar que en promedio las tasas de retiro son mayores entre las mujeres, no obstante, en el largo plazo se evidencia una convergencia en los patrones observados en las tasas de retiro de ambos sexos.

Por último, en 2013 se observa la disminución de los ingresos a la fuerza de trabajo, equiparándose a los retiros, dinámica relacionada por un lado con los bajos niveles de fecundidad registrados a principios del siglo XXI, y por otro, al aumento de la esperanza de vida. Todo ello finalmente redunda en el envejecimiento de la estructura de la población magallánica así como de su fuerza de trabajo.

\section{Vacantes y reemplazo de la fuerza de trabajo magallánica}

El reemplazo de la población económicamente activa hace referencia a la capacidad de reponer satisfactoriamente aquellos trabajadores que se retiran

11 Este ejercicio es similar al reemplazo generacional de la población total, y sirve para conocer los volúmenes de ingreso y retiros del mercado de trabajo en perspectiva generacional. Este panorama general no excluye la
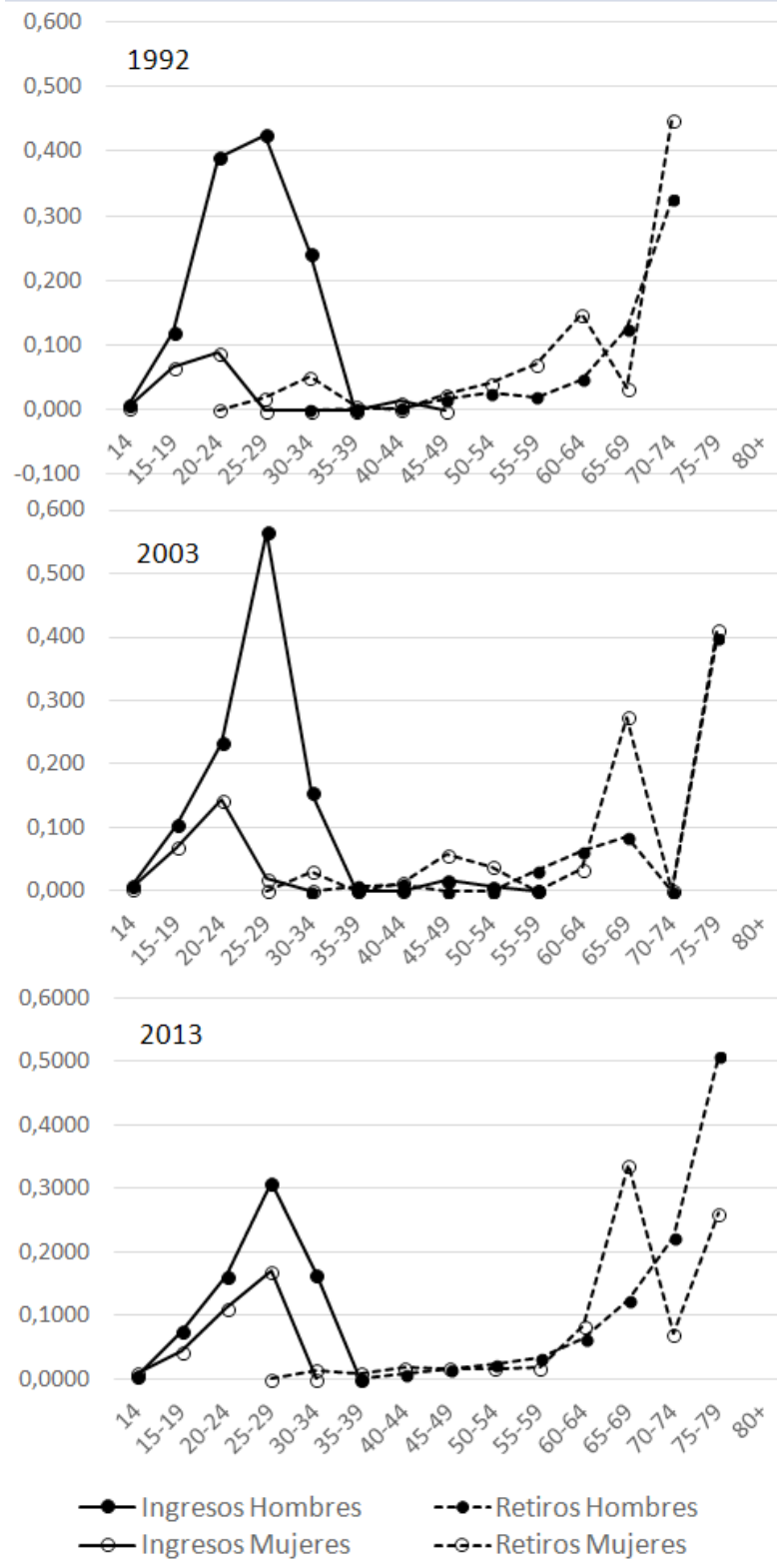

Fig. 7. Tasas de ingreso y retiro de la actividad, Magallanes 1992-2013.

Fuente: Elaboración propia.

del mercado de trabajo en un año determinado con nuevos trabajadores que se incorporen a la vida activa ${ }^{11}$. Las nuevas generaciones que

posibilidad de que pueda darse el caso de que en ciertos sectores de actividad se produzca un desequilibrio entre la oferta y la demanda de trabajadores para cubrir los puestos disponibles. 
se incorporan al mercado de trabajo en un año determinado encuentran trabajo no sólo en función de la expansión de la economía y de la creación de nuevos puestos de trabajo, sino también en función de las vacantes disponibles producto del retiro de la actividad, principalmente por motivo de la edad y de la jubilación (Livi-Bacci, 2012).

Es sabido que la capacidad de absorción de la mano de obra no corresponde a la demanda que origina el mero crecimiento económico, sino que del equilibrio entre la oferta y los requerimientos de la producción. En este sentido, el equilibrio se basa en dos fuentes, primero, la demográfica, respecto a la oferta de trabajo. En segundo lugar, la económica, que está sujeta, por un lado, a la coyuntura económica que afecta a la producción, y por otro lado, a cambios estructurales en la producción como consecuencia del progreso tecnológico, de los cambios en la orientación de consumo y de la política fiscal, los que son acompañados por cambios ocupacionales en el mismo sentido (Elizaga, 1979).

Como hemos visto, el progreso social y económico en la región, reflejado sobre el aumento de la esperanza de vida, señala que el papel jugado

Tabla 10. Razón de reemplazo de la fuerza de trabajo. Magallanes 1992-2013.

\begin{tabular}{ccc}
\hline & Hombres & Mujeres \\
\hline 1992 & 96 & 96 \\
2003 & 97 & 80 \\
2013 & 86 & 67 \\
\hline
\end{tabular}

Fuente: Elaboración propia.

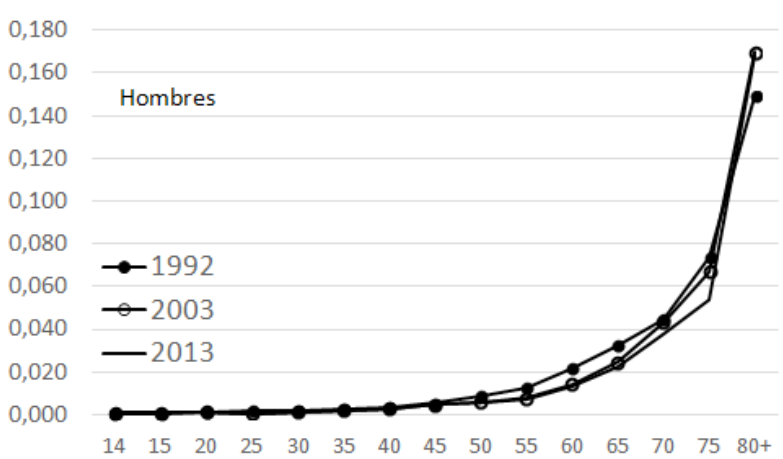

por la mortalidad en la dinámica de la fuerza de trabajo magallánica ha sido la reducción del número relativo de trabajadores nuevos requeridos para llenar las vacantes producidas por las defunciones, tendencia que se intensifica a medida que transcurre el siglo XXI (Fig. 8).

A pesar de ello, la razón de reemplazo ha mejorado entre 1992 y 2013, detrás de esta tendencia se encuentra la disminución del ingreso a la fuerza de trabajo (Tabla 10).

En 1992 por cada 100 retiros se producían 96 ingresos en ambos sexos, escenario que dejaba poco espacio a vacancias y que convierte en fundamental la creación de nuevas fuentes de trabajo. Dos décadas después la situación es distinta, apreciándose una mayor holgura en cuanto al reemplazo sobre todo en las mujeres. Detrás de esta tendencia está, por un lado, la disminución de los ingresos a la fuerza de trabajo dados los bajos niveles de fecundidad. Por otro lado, este último escenario cuenta con dos interpretaciones. Primero, da cuenta de una situación a priori beneficiosa para el empleo de las nuevas cohortes que ingresan al mercado con mayores oportunidades de empleabilidad; segundo, da cuenta de una oferta de trabajo insuficiente para cubrir los puestos de trabajo que quedan vacantes, tendencia que se espera se profundice a medida que avance el envejecimiento.

\section{CONCLUSIONES}

Con perspectiva del largo plazo, observamos que en Magallanes los dos agentes principales del cambio demográfico, las tendencias de mortalidad y fecundidad, están marcados por el declive.

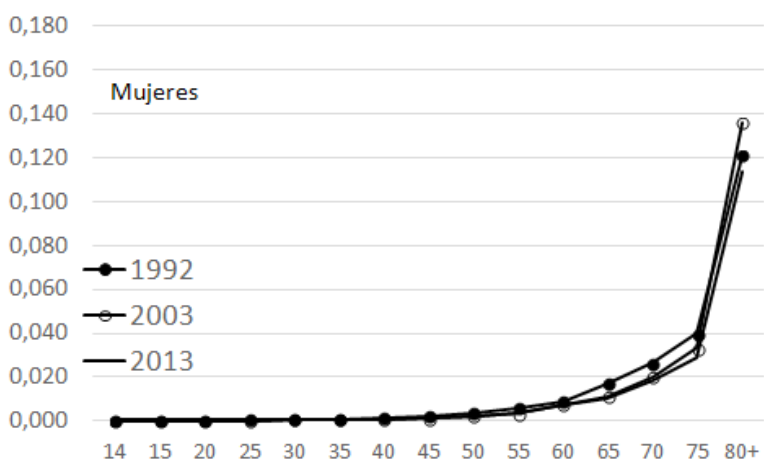

Fig. 8. Tasas de mortalidad de la fuerza de trabajo. Magallanes 1992-2013.

Fuente: Elaboración propia. 
Por otro lado, aunque en general la historia de la TD en Magallanes encaja dentro de las etapas a nivel nacional, se advierten diferencias de niveles y de tendencias, como sucede con la fecundidad durante las décadas de 1950 y 1960, que posicionan a la región entre las pioneras del cambio demográfico experimentado a nivel país. De esta manera, en la primera parte de este artículo hemos podido comprobar los cambios en la estructura de la población de la región desde el inicio de la TD, los que se sintetizan en un proceso de cambio demográfico dominado por el paulatino envejecimiento, que adquiere mayor intensidad hacia finales del siglo $\mathrm{XX}$ y principios del siglo XXI.

Dentro de este contexto la población en edad de trabajar o potencialmente productiva de la región ha experimentado cambios tanto en su tamaño como en sus características, lo que está estrechamente relacionado con su participación económica. En este sentido, las tendencias observadas en las tasas de participación económica por edades mostradas por la región entre los años 1992 y 2013 comprueban, primero, la reducción en las edades de entrada a la actividad económica -entre los 15 y 24 años-. Segundo, el aumento en las edades intermedias -entre los 25 y 49 años-, aquellas de mayor potencial productivo. Tercero, el aumento de la participación en las edades avanzadas, cercanas a la edad legal de retiro. Tendencias que están presentes tanto en hombres como en mujeres. En el primer y segundo caso, la postergación en el ingreso a la fuerza de trabajo como la mayor participación en edades intermedias, se explican por el aumento del nivel educativo y de capacitación de las nuevas cohortes que ingresan a la fuerza de trabajo. En el tercer caso, la tendencia creciente de participación económica a edades avanzadas, se explica por la mayor esperanza de vida, la cual incluso permite la permanencia en la actividad más allá de la edad legal de retiro. Por último, la participación de las mujeres experimenta un aumento significativo en todas las edades.

A raíz de estos cambios ocurridos en la fuerza de trabajo, y como hemos comprobado a través de las TVA, los cambios más importantes en cuanto a la duración de la vida activa entre 1992 y 2013 se sintetizan en, primero, el aumento de los años netos de vida activa con que cada trabajador contribuye a la producción a lo largo de su vida entre 1992 y 2013. Segundo, tanto el patrón de ingreso como de retiro de la fuerza de trabajo están marcados por la postergación. Tercero, los años potenciales de actividad, medidos a través de la EVA al inicio de la vida económica -15 años-, muestran un aumento entre 1992 y 2013 a pesar de la reducción de la participación económica en las edades de entrada.

De esta manera, en cuanto al aumento en la duración de la vida activa destacan dos hechos importantes. Primero, el aumento de la duración se da gracias a la postergación del retiro. La reducción de la mortalidad y el consecuente aumento de la esperanza de vida, reducen los retiros anticipados de vida activa, y por otro lado, hacen posible, ya sea por necesidad o voluntad, que se extienda la actividad más allá de los 65 años. En segundo lugar, el aumento de la EVA es mayor en las mujeres que en los hombres. Además del aumento de la esperanza de vida en las mujeres, el aumento generalizado del nivel educativo de la población magallánica, es un hecho estrechamente relacionado con la mayor incorporación de la mujer al mercado de trabajo, aumentando así significativamente la EVA. En este sentido podemos afirmar que entre 1992 y 2013, a pesar del retraso en el inicio de la actividad económica, los efectos del envejecimiento sobre la fuerza de trabajo magallánica compensan los años perdidos al inicio gracias a la postergación en el retiro.

En este trabajo hemos podido comprobar las transformaciones tanto en la estructura demográfica de la fuerza de trabajo, así como las transformaciones económicas derivadas de la coyuntura económica observadas a partir del periodo 1992-2013. Durante este periodo, se comprueba los efectos combinados de una estructura demográfica en la que abunda la oferta de mano de obra potencial, que a su vez muestra un crecimiento en su participación efectiva dando como resultado el aumento de la EVA. Respecto a los desafíos planteados por el envejecimiento hacia el futuro cabe señalar que, si bien, más años de vida activa pueden entenderse como un efecto positivo, éstos deben ir acompañados por un mercado de trabajo con capacidad para absorber de forma productiva la creciente oferta de trabajo, de lo contrario, los efectos positivos que implican el retroceso de la mortalidad, se transformarán en un obstáculo al reemplazo generacional de la fuerza de trabajo. 


\section{AGRADECIMIENTOS}

Agradecimientos al proyecto POSTDOC_ DICYT, Código 031762LLJ_POSTDOC.

\section{BIBLIOGRAFÍA}

\section{Referencias Bibliográficas}

Andersson, B. (2001). Scandinavian evidence on growth and the age structure. Regional Studies, 35(5), 377-390.

Behm, H. (2014). Los determinantes de la mortalidad y las diferencias socioeconómicas de la mortalidad en la infancia. Población y Salud en Mesoamérica, 12(1), 1-9.

Bloom, D., \& Williamson, J. (1998). Demographic transition and economic miracles in emerging Asia. The World Bank Economic Review, 12(3), 419-455.

Bloom, D. E., Canning, D., \& Sevilla, J. (2001). Economic growth and the demographic transition. Working Paper (8685), National Bureau of Economic Research.

Bloom, D. E., Canning, D., \& Sevilla, J. (2003). The demographic dividend: A new perspective on the economic consequences of population change. RAND.

Bloom, D. E., Canning, D., \& Sevilla, J. (2004). The effect of health on economic growth: a production function approach. World development, 32(1), 1-13.

Chackiel, J. (2004). La dinámica demográfica en América Latina. Serie de Población y Desarrollo(52). Santiago de Chile: Centro Latinoamericano y Caribeño de Población (CELADE).

Cerda, R. (2008). Cambios demográficos y sus impactos en Chile. Estudios públicos, 110, 89-164.

Cornejo Chávez, R. (2006). El experimento educativo chileno 20 años después: una mirada crítica a los logros y falencias del sistema escolar. REICE. Revista Iberoamericana sobre Calidad, Eficacia y Cambio en Educación, 4(1).

Devolder, D., Nos, R., \& Panareda, E. (2006). La fecundiad de las generaciones españolas nacidas en la primera mitad del siglo XX: un estudio a escala provincial. Revista de Demografía Histórica, 24(1), 57-90.

Donoso, E., \& Carvajal, J. (2009). Reducción de la fecundidad y envejecimiento de la población de mujeres chilenas en edad fértil: 1990-2004. Revista médica de Chile, 137(6), 766-733.

Easterlin, R. (1978). What will 1984 be like? Socioeconomic implications of recent twits in age structure. Demography, 15(4), 397-432.

Elizaga, J. (1979). Dinámica y economía de la población. Santiago de Chile: Centro Latinoamericano y Caribeño de Población (CELADE).
Galleguillos, S., \& Sierralta, M. I. (1989). Determinantes de la mortalidad de la población chilena en Chile. Estudios de economía, 16(2), 224-247.

García-Moro, C., Hernández, M., \& Esparza, M. (2015). Patrones de mortalidad y supervivencia en la población colonizadora de Magallanes (1885 1920). Magallania, 43(2), 45-56.

Gutiérrez de Mesa, J. (2010). Evolución de la actividad y el paro en España y sus regiones en el periodo 2005-2010 a través de la esperanza de vida laboral en ausencia de mortalidad. $7^{a}$ Workshop-APDR. XXXVI reunión de Estudios Regionales-AECR. Badajoz-Elvas.

Hernández, M., García-Moro, C., \& Martinic Beros, M. (1998). Evolución demográfica de la población de Tierra del Fuego (Región de Magallanes). Anales del Instituto de la Patagonia, 26, 41-68

Hernández, M., García-Moro, C., \& Esparza, M. (2015). Edad materna y paridad en la población de Tierra del Fuego a mediados del siglo XX: Comparación con la Isla de Pascua. Magallania, 43(2), 57-70.

Hernández, H. (1994). La Población Chilena. La población chilena: dinámica demográfica, prospectiva y problemas. Revista Tiempo y Espacio, 4.

Hernández, H. (2015). La poblacion chilena: dinámica demográfica, prospectiva y problemas. Tiempo y Espacio, (4), 8-36.

Hayward, M., \& Grady, W. (1990). Work and retirement amog a cohort of older men in the United States, 1966-1983. Demography, 27(3), 337-356.

Instituto Nacional de Estadísticas (Chile) (2004). La Mortalidad en Chile según las Tablas Abreviadas de Mortalidad, por sexo, país y regiones 1919-2002. Santigo de Chile: INE.

Larrañaga, O. (2006). Participación laboral de la mujer en Chile 1958-2003. Universidad de Chile, Departamento de Economía, Santiago de Chile.

Livi-Bacci, M. (2012). Historia mínima de la población mundial. Barcelona. Ariel.

Martínez Pizarro, J. (1998). La transición demográfica y las diferencias sociales de la fecundidad y la mortalidad infantil en Chile. Santiago de Chile: MIDEPLAN.

Martinic, M. (2002). Breve historia de Magallanes. Punta Arenas, Chile.

Martinic, M. (2004). La minería del carbón en Magallanes entre 1868-2003. Historia, 37(1), 129-167

Martinic, M. (2006). El poblamiento rural en Magallanes durante el siglo XX: realidad y utopía. Magallania, 34(1), 5-20.

Martinic, M. (2016) Reescribiendo la historia: Algunas reflexiones sobre el conocimiento y dominio del territorio nororiental de Magallanes (1870-1900). Magallania, 44(2), 
5-36.

Medina, E., \& Kaempffer, A. (2000). Mortalidad del adulto en Chile. Revista médica de Chile, 128(10), 1144-1149.

Mella, O. (2003). 12 años de Reforma Educacional en Chile. Algunas consideraciones en torno a sus efectos para reducir la inequidad. REICE. Revista Iberoamericana sobre Calidad, Eficacia y Cambio en Educación, 1(1).

Pardo, L. (1979). Efectos de las variaciones económicas en la fecundidad: Chile 1952-1980. Estudios de Economía, 6(2), 75-132.

Partida-Bush, V. (1996). Tabla de Vida Activa. México, D.F.: COLMEX.

Partida-Bush, V. (2000). Cambios en el mercado laboral urbano medidos a través de la esperanza de vida activa. Papeles de Población, 6(26).

Rivero, R., \& Spijker, J. (2015). Del rejuvenecimiento al envejecimiento de la población ¿o viceversa?: Chile en el contexto de América Latina, 1950-2050. Notas de Población, 42(101), 127-155.

Riesco, M. (2006). Tres exigencias mínimas para la reforma previsional. Documento En Foco, 67, 1-15

Riesco, M. (2009). El modelo social chileno comienza a cambiar. Revista internacional del Trabajo, 128(3), 311-330.

Reher, D. (1994). El pasado demográfico de América Latina: una cuestion de perspectivas. En ABEP, y otros (Eds.), La transición demográfica en América Latina y el Caribe (pp. 98-109). Ciudad de México: IV Conferencia Latinoamericana de Población.

Reher, D. (2004). The demographic transition revisited as a global process. Population Space and Place, 10(1), 19-41.

Rosero-Bixby, L., \& Jiménez Fontana, P. (2012). Retos y oportunidades del cambio demográfico para la política fiscal. San José de Costa Rica: Centro Centroamericano de Población de la Universidad de Costa Rica.

Saad, P. M., Miller, T., Holz, M., \& Martínez, C. (2012). Juventud y bono demográfico en Iberoamérica. Madrid: Organización Iberoamericana de Juventud (OIJ), Centro Latinoamericano y Caribeño de Demografía (CELADE)División de Población de la Comisión Económica para América Latina y el Caribe (CEPAL) y Fondo de Población de las Naciones Unidas UNFPA.

Ruiz F. J. (2006) Estudio básico investigación comportamiento migratorio población XII Región. Punta Arenas: Instituto Nacional de Estadísticas (INE).

Szot Meza, J. (2003). La transición demográfico-epidemiológica en Chile 1960-2001. Revista Española de Salud Pública, 77(5), 605-613.

Vera, J. (2008). Magallanes: Dinámica Económica y Demográfica 1960-2006; Leyes de Excepción para el Desarrollo; qué hacer y qué evitar. Magallania, 36(2), 63-78.

Villa, M., \& González, D. (2004). Dinámica de la demográfica de Chile y América Latina: Una visión a vuelo de pájaro. Revista de Sociología, 18.

Weilti, C. (1997). Demografía I. México D.F.: Universidad Nacional Autonoma de México.

\section{Fuentes Primarias}

Instituto Nacional de Estadísticas (Chile) (1964). XIII Censo de Población y II de Vivienda (1960). Santiago de Chile: Dirección de Estadísticas y Censos, Ministerio de Economía.

Instituto Nacional de Estadísticas (Chile) Anuarios Estadísticos 1920-1951. Santiago de Chile: Dirección de Estadísticas y Censos, Ministerio de Economía.

Instituto Nacional de Estadísticas (Chile) Anuarios de Demografía 1952-1999. Santiago de Chile: Dirección de Estadísticas y Censos, Ministerio de Economía.

Instituto Nacional de Estadísticas (Chile) Anuarios de Estadísticas Vitales 2000-2014. Santiago de Chile: Dirección de Estadísticas y Censos, Ministerio de Economía.

Instituto Nacional de Estadísticas (Chile) (2014b). Actualización de población 2002-2012 y proyecciones 2013-2020. Dirección de Estadísticas y Censos, Ministerio de Economía.

http://www.ine.cl/canales/chile_estadistico/familias/ demograficas_vitales.php [última visita junio de 2017].€ Integrated Public Use Microdata Series International (IPUMS-I). Microdatos Censales:

https://www.ipums.org/ [última visita junio de 2017].

Ministerio de Desarrollo Social, Encuesta de Caracterización Socioeconómica Nacional (Casen)http://observatorio. ministeriodesarrollosocial.gob.cl/casen/casen_obj.php [última visita junio de 2017]. 
ANEXO Tabla A.1. Tablas de Vida Activa. Región de Magallanes y la Antártica Chilena 1992-2013.

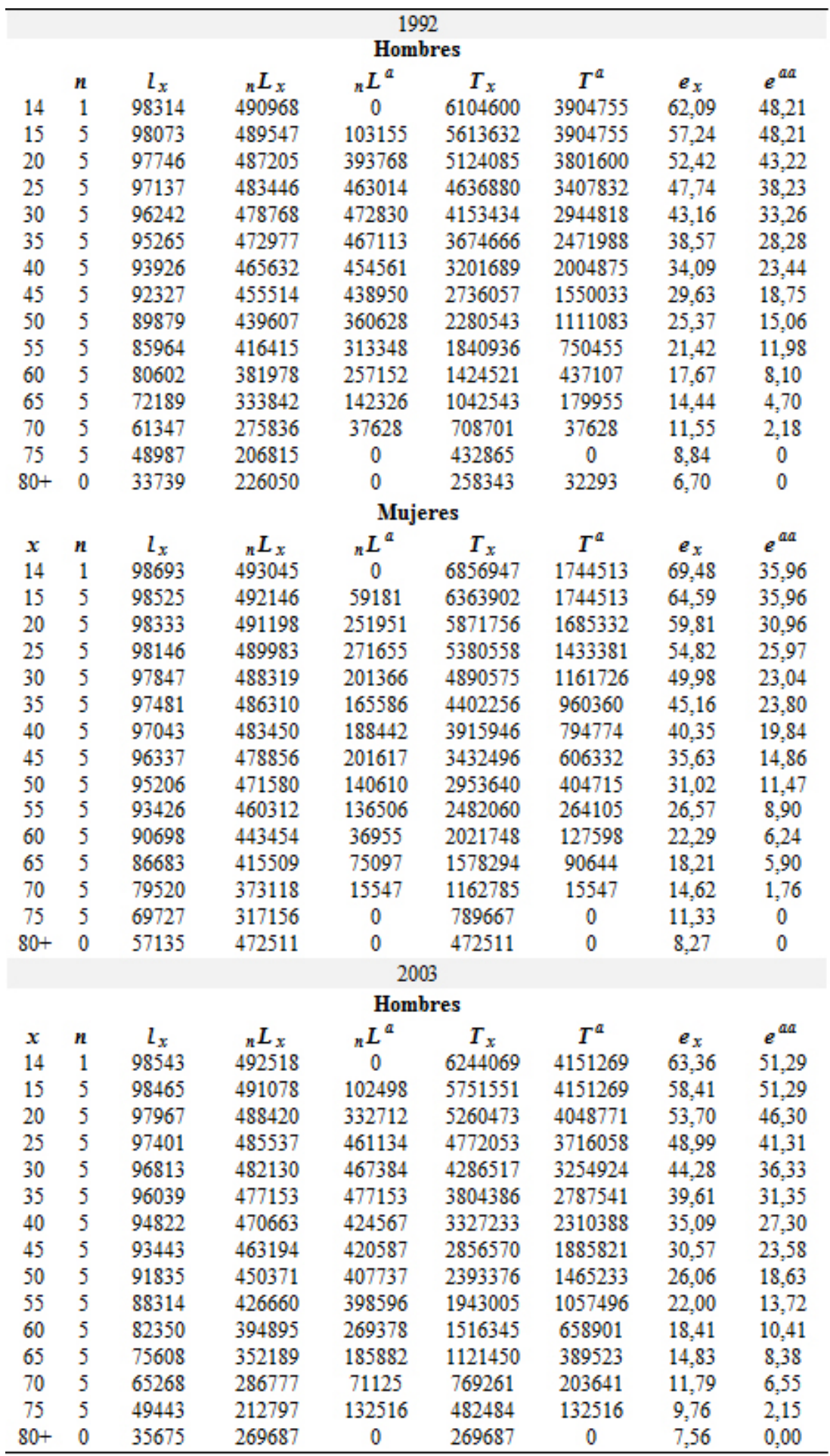


Tabla A.1. Continuación.

\begin{tabular}{|c|c|c|c|c|c|c|c|c|}
\hline \multicolumn{9}{|c|}{ Mujeres } \\
\hline$x$ & $n$ & $l_{x}$ & ${ }_{n} L_{x}$ & ${ }_{n} L^{a}$ & $T_{x}$ & $T^{a}$ & $e_{x}$ & $e^{a a}$ \\
\hline 14 & 1 & 98899 & 494116,54 & 0 & 6909164 & 2667055 & 69,86 & 42,22 \\
\hline 15 & 5 & 98748 & 493233 & 55218 & 6415048 & 2667055 & 64,96 & 42,22 \\
\hline 20 & 5 & 98546 & 491900 & 266305 & 5921815 & 2611837 & 60,09 & 37,23 \\
\hline 25 & 5 & 98215 & 489951 & 377522 & 5429915 & 2345532 & 55,29 & 32,24 \\
\hline 30 & 5 & 97766 & 488062 & 289157 & 4939964 & 1968010 & 50,53 & 27,25 \\
\hline 35 & 5 & 97459 & 486306 & 287570 & 4451902 & 1678853 & 45,68 & 25,68 \\
\hline 40 & 5 & 97063 & 483746 & 338746 & 3965596 & 1391283 & 40,86 & 20,69 \\
\hline 45 & 5 & 96436 & 479670 & 246185 & 3481850 & 1052536 & 36,11 & 16,76 \\
\hline 50 & 5 & 95432 & 472719 & 195053 & 3002180 & 806351 & 31,46 & 15,89 \\
\hline 55 & 5 & 93655 & 462298 & 167083 & 2529462 & 611298 & 27,01 & 13,38 \\
\hline 60 & 5 & 91264 & 443516 & 270185 & 2067164 & 444215 & 22,65 & 8,44 \\
\hline 65 & 5 & 86142 & 416745 & 94229 & 1623648 & 174030 & 18,85 & 4,55 \\
\hline 70 & 5 & 80556 & 381200 & 0 & 1206902 & 79801 & 14,98 & 6,99 \\
\hline 75 & 5 & 71924 & 324304 & 79801 & 825702 & 79801 & 11,48 & 2,25 \\
\hline $80+$ & 0 & 57797 & 501399 & 2558 & 503957 & 2558 & 8,68 & 0,00 \\
\hline \multicolumn{9}{|c|}{2013} \\
\hline \multicolumn{9}{|c|}{ Hombres } \\
\hline$x$ & $n$ & $l_{x}$ & ${ }_{n} L_{x}$ & ${ }_{n} L^{a}$ & $T_{x}$ & $T^{a}$ & $e_{x}$ & $e^{a a}$ \\
\hline 14 & 1 & 99251 & 495292 & 0 & 4062197 & 2388223 & 64,99 & 51,48 \\
\hline 15 & 5 & 98866 & 493309 & 70868 & 4062197 & 1892932 & 60,23 & 51,48 \\
\hline 20 & 5 & 98458 & 490782 & 268201 & 3991328 & 1470491 & 55,47 & 46,49 \\
\hline 25 & 5 & 97855 & 488439 & 428394 & 3723127 & 1247909 & 50,80 & 41,51 \\
\hline 30 & 5 & 97520 & 486028 & 449862 & 3294734 & 1187864 & 45,97 & 36,52 \\
\hline 35 & 5 & 96891 & 482272 & 482272 & 2844872 & 1151698 & 41,25 & 31,53 \\
\hline 40 & 5 & 96018 & 476791 & 449241 & 2362600 & 1151698 & 36,60 & 26,56 \\
\hline 45 & 5 & 94698 & 467992 & 453602 & 1913359 & 1124149 & 32,08 & 22,09 \\
\hline 50 & 5 & 92498 & 456238 & 400797 & 1459757 & 1109759 & 27,78 & 17,82 \\
\hline 55 & 5 & 89997 & 441762 & 380161 & 1058960 & 1054317 & 23,48 & 13,95 \\
\hline 60 & 5 & 86708 & 419536 & 302481 & 678800 & 992716 & 19,28 & 10,00 \\
\hline 65 & 5 & 81106 & 383922 & 225707 & 376318 & 875661 & 15,44 & 6,76 \\
\hline 70 & 5 & 72463 & 331186 & 77964 & 150611 & 717445 & 11,98 & 3,98 \\
\hline 75 & 5 & 60012 & 264480 & 72647 & 72647 & 464223 & 8,95 & 2,20 \\
\hline $80+$ & 0 & 45780 & 272390 & 15693 & 15693 & 272390 & 5,95 & 0,00 \\
\hline \multicolumn{9}{|c|}{ Mujeres } \\
\hline$x$ & $n$ & $l_{x}$ & ${ }_{n} L_{x}$ & ${ }_{n} L^{a}$ & $T_{x}$ & $T^{a}$ & $e_{x}$ & $e^{a a}$ \\
\hline 14 & 1 & 99118 & 495588,96 & 15285,069 & 2748997 & 4444198 & 72,57 & 43,66 \\
\hline 15 & 5 & 99118 & 495480 & 52795 & 2733712 & 3963894 & 67,57 & 43,66 \\
\hline 20 & 5 & 99074 & 495158 & 185131 & 2680917 & 3521210 & 62,60 & 38,66 \\
\hline 25 & 5 & 98989 & 494729 & 397767 & 2495786 & 3211183 & 57,65 & 33,66 \\
\hline 30 & 5 & 98903 & 493728 & 348629 & 2098019 & 3114221 & 52,70 & 28,66 \\
\hline 35 & 5 & 98589 & 491893 & 345693 & 1749390 & 2969121 & 47,86 & 25,72 \\
\hline 40 & 5 & 98168 & 489856 & 312780 & 1403698 & 2822921 & 43,05 & 21,78 \\
\hline 45 & 5 & 97774 & 487306 & 284835 & 1090918 & 2645845 & 38,22 & 18,68 \\
\hline 50 & 5 & 97148 & 482861 & 259208 & 806082 & 2443374 & 33,45 & 15,18 \\
\hline 55 & 5 & 95996 & 476086 & 235511 & 546875 & 2219721 & 28,82 & 11,33 \\
\hline 60 & 5 & 94438 & 463959 & 208597 & 311364 & 1979146 & 24,25 & 7,18 \\
\hline 65 & 5 & 91145 & 444115 & 49179 & 102767 & 1723784 & 20,04 & 4,21 \\
\hline 70 & 5 & 86501 & 413371 & 35222 & 53588 & 1328848 & 15,98 & 6,53 \\
\hline 75 & 5 & 78847 & 367503 & 18366 & 18366 & 950698 & 12,29 & 3,45 \\
\hline $80+$ & 0 & 68154 & 601562 & 3715 & 3715 & 601562 & 8,83 & 0,00 \\
\hline
\end{tabular}

Fuente: Elaboración propia. 
